\title{
A Load Sharing Principle in Abdominal Wall Reconstruction. Communication and Collaboration Among Plastic \& Reconstructive Surgeons, Oncologic Surgeons, and General Surgeons.
}

Brian P Dickinson ( $\boldsymbol{\nabla}$ drdickinson@drbriandickinson.com )

Hoag Memorial Presbyterian Hospital https://orcid.org/0000-0001-7185-0860

\section{Monica Vu}

University of California Riverside

Nikkie Vu-Huynh

University of California Los Angeles

\section{Alexander Shadid}

Hoag Memorial Presbyterian Hospital

\section{Todd S Harris}

Hoag Memorial Presbyterian Hospital

Robert Selby

Hoag Memorial Presbyterian Hospital

\section{Larry Perl}

Hoag Memorial Presbyterian Hospital

\section{Kristina Mori}

Hoag Memorial Presbyterian Hospital

\section{Peter Wang}

Hoag Memorial Presbyterian Hospital

\section{Steven Beanes}

Hoag Memorial Presbyterian Hospital

Alberto Mendivil

Hoag Memorial Presbyterian Hospital

Michael Hurwitz

Hoag Memorial Presbyterian Hospital

\section{Technical advance}

Keywords: Ventral Hernia, Recurrent Hernia, Abdominal Wall Reconstruction, Component Separation, Acellular Dermal Matrix 
Posted Date: September 24th, 2020

DOl: https://doi.org/10.21203/rs.3.rs-58066/v1

License: (c) (i) This work is licensed under a Creative Commons Attribution 4.0 International License. Read Full License 


\section{Abstract}

Background: Abdominal wall reconstruction of ventral hernias can be challenging. Patients have undergone successful treatment of cancer or other intra-abdominal processes and have worked hard to get back to a "normal" life. Successful repair of abdominal wall hernias can be difficult as many comorbidities persist. Previous radiation, ongoing smoking, or overall protein malnutrition may affect wound healing and overall outcome. Surgical teams need to communicate to maximize repair success and decrease recurrence.

Purpose: To create a systematic approach from our current method of abdominal wall reconstruction that facilitates communication between general surgeons and plastic and reconstructive surgeons for optimizing hernia repair outcomes.

Methods: A retrospective chart review was done on patients who underwent abdominal wall reconstruction of ventral hernias and recurrent hernias with component separation and placement of strattice acellular dermal matrix or synthetic mesh over a 10 year period. Pre-op imaging consisted of an abdominal/pelvic CT-scan. A surgical flight plan was created to determine a plane of Strattice insertion and abdominal wall exposure. Pre/post-operative CT-scans were compared as well as before and after photographs. Patient satisfaction was assessed subjectively in follow-up appointments.

Results: The most commonly encountered clinical scenarios were placed into a pictorial essay and a step by step approach to abdominal wall reconstruction was then created. Strattice or synthetic mesh were placed using a "load-sharing" principle and restoration of a dynamic abdominal wall was associated with high patient satisfaction, a more functional repair, and a lower incidence of recurrence.

Conclusions: Successful repair of primary and recurrent abdominal wall hernias requires communication between general surgeons and plastic \& reconstructive surgeons. Incision placement is important for adequate exposure and the ability to place transfascial sutures. T-junction skin breakdown and seroma formation can be minimized by maintaining blood supply and minimizing dead space. High protein nutritional stores are important for expeditious healing.

\section{Background}

Abdominal wall reconstruction and hernia repair surgery continue to evolve with the intention to successfully repair the abdominal wall, restore muscular dynamics, and minimize recurrences. This trio of expectations is now being comprehensively defined as "Abdominal Core Health" and has many physical and mental benefits.[1, 2] Repair of primary and recurrent abdominal wall hernias with component separation and Strattice acellular dermal matrix is a technique where plastic surgeons and general surgeons work closely together to close abdominal walls and optimize functional as well as aesthetic outcomes reestablishing abdominal core health. $[3,4]$ There are many different locations of hernias encountered by the general surgeon and plastic surgeon team: ventral, inguinal, umbilical, parastomal as well as the lateral abdominal wall (flank).[5] Hernias are prevalent in the population and their presence is 
not inconsequential.[6-8] In fact, hernia repair has become one of the most common conditions that general surgeons encounter.[9-10] As the population ages, and as comorbidities such as obesity, pulmonary disease, DVT, cardiac disease, and recurrent hernias become more prevalent, the complexity of hernia repairs and their post operative management increases.

Abdominal wall reconstruction is challenging and humbling for both patients and surgeons and complications can occur with both prosthetic and biologic mesh.[11-15] Often, patients have undergone successful treatment of their cancer or other intra-abdominal process and have worked hard to get back to a "normal life". A hernia can be cumbersome for the patient and become both a physical and psychological "ball and chain". Successful repair, which requires maintaining a sound long-term repair, can be difficult as many comorbidities persist. General surgeons and plastic and reconstructive surgeons should communicate the anatomic and physiologic obstacles they foresee after history and examination of the patient to maximize repair success and decrease recurrence. This effective communication between the operating surgeons and review of CT-scans can help formulate successful flight plans for surgery. The more time spent in the pre-operative preparation and in the flight planning stage, the more optimized the patient is for success even in the face of previous radiation, comorbidities, or encountered post-operative obstacles.

The plastic and reconstructive surgeon author of this paper (BD) is thankful for everything he has learned from the general and oncologic surgeons in this manuscript. The impetus for writing this paper comes from early humbling experience with hernia recurrences or failure to achieve completely satisfied patients. Early repairs of abdominal wall hernias and their recurrences came from a standard "load bearing" approach to ventral hernia repair [Diagram 1]. Our experience was similar to others in the literature with "bridged" repairs.[16-17] In our previous "load bearing" repairs, patients would recur or would not be completely satisfied with the contour of their abdomen as they had a persistent bulge and often back pain would persist as a result of an adynamic core. As the first authors' experience with repair of facial fractures and free microvascular fibula flap reconstructive procedures increased and as the first author learned more from the general surgeon and oncologic authors, we chose to adopt the "load sharing" principle of fracture repair to abdominal wall hernia repair. The Arbeitsgemeinschaft für Osteosynthesefragen (AO), an organization formed to conduct research in bone healing helped to explain a better way to repair the abdominal wall defect that could potentially be longer lasting in the patients with high BMI except that the forces were not compressive bone forces but rather contractile forces. [Diagram 1] [AO Reference] In mandible fractures the plate can "bear the load" of the forces on the mandible, but an alternative construct often needed for larger bones is the "load sharing" principle that allows the bone to begin to withstand the more of the compressive forces. We tried to learn, study, and adapt this "load sharing principle" to large abdominal wall reconstructions. The only difference is the vector of the forces. [Diagram 2]

The predominant load sharing materials in our experience of abdominal wall reconstruction is Strattice acellular dermal matrix. This was usually chosen given the thickness and tensile strength that was greater than human matrices [reference]and consistent with the literature. Strattice was also generally 
chosen because of strength and biologic nature and safety with a lower risk of infection and more easily to manage complications in a higher risk population of patients who may be at higher risk given their previous cancer, infectious abdominal processes, or additional comorbidities in our abdominal wall reconstruction population.[18-20] Synthetic mesh is a viable option in patients with stable soft tissue and lower risk of infectious complications. The authors have used both with good outcomes. We present here a step by step approach with examples of complex abdominal wall reconstruction for an audience of residents, fellows, or plastic and general surgeons teams who choose to embark on abdominal wall reconstruction.

\section{Methods}

A retrospective chart review was conducted on all patients who underwent abdominal wall reconstruction with component separation and Strattice acellular dermal matrix or synthetic mesh in retro rectus and intraperitoneal spaces by the authors. Charts were reviewed and categorized by the most commonly presenting clinical scenarios and then surveyed for the most common challenging situations affecting the abdominal wall repair. Before and after photos were examined and charts were reviewed for complications. A step by step approach was created to convey to general surgeons and plastic and reconstructive surgeons, an organized management approach to these common problems. Classical examples of the more commonly encountered difficult cases were analyzed pre-operatively, and postoperatively. Intra-operative methods and maneuvers were evaluated alongside their postoperative results.

The patients were seen pre-operatively by the general surgeon and plastic and reconstructive surgeon. Patients underwent CT of the abdomen and pelvis with contrast prior to surgery. Hernia size and the surrounding muscular health was identified by CT scan and then correlated with the physical exam findings. A tentative flight plan was then created by the general surgeon and plastic and reconstructive surgeon team after first discussing the case, and reviewing the CT-scan. Finally, the flight plan was then modified to include incision choice for hernia repair. The factors examined when creating the surgical incision flight plan include body mass index, size of hernia defect measured on CT-scan, location of hernia, amount of dead space that will be created by exposure, and medical comorbidities. Body mass index was counseled highly in patients to predict likelihood of post-operative wound complications. High protein diet was encouraged in all patients for 4 weeks before surgery and for 4 weeks after surgery.

When available, post-operative CT-scans were evaluated and compared to preoperative CT-scans. CTscans were usually completed if patients had symptoms of pain after trauma or exercise or any gastrointestinal symptoms or as part of their cancer surveillance.

All participants provided written consent for their use of photographs for presentation and publication. Patients are not identified by name in any photographs or text. Patients are aware that in some circumstances, the photographs may make their identity recognizable. In addition, patient identifying information has been removed from images and faces are omitted from photographs, irrelevant clinical information not helpful to understanding the hernia repair principles has been omitted. 


\section{Results}

Patient satisfaction rate was high among hernias repaired with the open approach, component separation and placement of acellular dermal matrix. The most common complications post-operatively were seroma and partial skin necrosis treated with early debridement and closure. In one year there was one episode of recurrence in a patient who underwent component separation without placement of dermal matrix. The patient had a high BMI secondary to muscle and was an avid weightlifter. Patients notice weight loss with the initiation of a high protein diet pre-operatively. A majority of patients continued the dietary changes post-operatively for more than eight weeks.

Group 1. Midline Ventral Hernias. Using the Midline Incision with or without the addition of Lower Abdominal Skin Flap

\section{Case 1}

[Figure 1a, 1b, 1c]

\section{Case 2}

[Figure 2a, 2b, 2c]

Group 2. Midline Ventral Hernias in the Presence of Subcostal Hernias. Using the Midline Incision and Subcostal Incision.

Diagram 3. The Strattice was placed in the retro-rectus space. The components of the rectus sheath were repaired in layers. Posterior sheath followed by retro-rectus placement of the acellular dermal matrix, approximation of rectus muscle, and repair of anterior sheath.

\section{Case 3}

[Figure 3a, 3b, 3c]

\section{Case 4}

[Figure 4a, 4b, 4c]

\section{Case 5}

[Figure 5a, 5b, 5c]

Group 3. Midline Ventral Hernias. Using Lower Abdominal Incisions in Presence of Previous Lower Abdominal Horizontal Incisions.

\section{Case 6}

$[6 a, 6 b, 6 c]$ 
Group 4, Patients in which a significant amount of skin will need to be "re-drapped" to maintain stable skin coverage over the hernia repair or "resected" to prevent the occurrence of a seroma.

\section{Case 7}

$[7 \mathrm{a}, 7 \mathrm{~b}]$

\section{Case 8}

$[8 \mathrm{a}, 8 \mathrm{~b}]$

Case 9

[Figure 9a, Fig. 9b]

Case

0. [Figure 10a, Fig. 10b]

Group 4. Midline Ventral Hernias in the Presence Previous lleostomy/Colostomy Hernias.

Case 11

[Figure 11a, 11b, 11c,]

Case 12

[Figure 12a, 12b, 12c, 12d, 12e, 12f]

Group 5. Midline Ventral Hernias in the Presence Previous lleostomy/Colostomy Hernias.

Case 13

[Figure 13a, 13b, 13c]

Group 6. Midline Ventral Hernias with loss of abdominal domain and herniation of small bowel contents in subcutaneous position.

\section{Case 14}

[Figure 14a, 14b, 14c]

Group 6. Ventral Hernias in tall muscular patients with high BMI.

\section{Case 15}

[Figure 15a, 15b, 15c, 15d] 


\section{Case 16}

[Figure 16a, 16b, 16c]

Group 7. Patients who chose to undergo repair without Bioprosthetic or Synthetic Mesh.

\section{Case 17}

[Figure 17a, 17b]

\section{Discussion}

Recurrent hernias and complex abdominal wall hernias are challenging cases. Patients have overcome challenging life obstacles of cancer or another intra-abdominal process. The patient may have become frustrated with its recurrence or development. Unfortunately, many of these patients present with ongoing or recurrent comorbidities which need to be controlled or optimized prior to embarking on abdominal wall reconstruction. It is important for members of the general surgery and plastic \& reconstructive team to have an understanding of each other's roles, approaches, and concerns, as well as how treatment decisions impact the patient's overall abdominal core health and aesthetic results.

We found that when planning these complex abdominal wall repairs, following a systematic method optimizes the likelihood that the procedure is performed safely and maximizes restoration of abdominal core health and aesthetic outcomes. We also plan with the intention to help patients understand the process and with the education that minor complications such as seroma or small wound dehiscence are commonplace given their challenging problem.

\section{Step 1. Where is the location of the hernia on the abdominal wall ? Are there multiple hernias? Is the hernia close to the umbilicus and can the umbilicus remain viable with undermining or need to be translocated?}

Invariably, the first step in the examination of a hernia patient is an examination of the CT-scan. When patients are referred to us, often their CT-scans arrive in the mail before the patients and/or we review the CT-scan of the abdomen on the computer prior to seeing the patient in the exam room. The important information to be gathered is the dimensions of the hernia, usually the widest part, and then the location. Hernias close to the umbilicus, while large can often be easily closed as there is a greater degree of laxity of the abdominal wall. There is less laxity at the costal margin and at the location of the iliac crests.

If multiple hernias are present, this may prevent the excursion of the abdominal wall and limit approximation of healthy tissue and closure. It is helpful to understand these dimensions and possibilities pre-operatively. Repairing lateral defects or subcostal defects with acellular dermal matrix or individual component separation may inhibit closure of a midline defect as there is now less laxity. This should be accounted for and may leave a midline abdominal wall defect with a bridged repair that is not dynamic. This may increase recurrence or even seroma formation post-operatively. 
Both the general surgeon and plastic and reconstructive surgeon need to know the location of the hernia relative to the umbilicus. The proximity of the hernia to the umbilicus may make keeping it very unrealistic as undermining may devascularized it. Keeping it on a widely undermined flap pedicle may create an unreasonable position, or maintaining it on the abdominal wall may devascularize it after the placement of muscular or transfascial sutures.

\section{Step 2. What are the physical examination findings?}

The physical examination commences after the standard history is taken in the office. During the physical examination, we ask the patient about their current height and weight. This is converted directly into BMI. The BMI is explained to the patient and their risks for postoperative complications such as seroma and wound dehiscence which are likely to occur but which will be addressed early or possibly later in the post-operative course, if appropriate.

During physical examination, we measure the hernia defect and then when applicable bring the ends of the hernia musculature together with the patient standing. This maneuver tends to be more difficult with the patient standing compared to when the patient is supine on the operating table against the effects of gravity.

After this point in the examination, the decision tree adjusts as to the incision choice and exposure used to gain access to the hernia or hernias.

\section{Step 3. Has the patient had a prior C-section scar, how lateral does the C-section scar extend, and does the patient have lower abdominal skin laxity? Has the patient had a prior abdominoplasty?}

Discerning early if the patient has a lower abdominal incision will often determine which incision pattern will be used. In the patient with a previous abdominoplasty or extended C-section the superficial inferior epigastric vessels or superficial circumflex epigastric have been divided and the lower abdominal skin will not remain viable if undermining is accomplished superior to this. In these cases, the abdominoplasty incision should be utilized or the extended c-section scar incorporated into the exposure. Assessing the distal viability of the skin in these cases is critical and in some cases there may be a need to return to the operating room in the postoperative period to freshen up wound edges and re-close the skin. This early potential return to the operating room can potentially prevent mesh, suture, or seroma colonization and a low threshold should be maintained for a return to the operating room for skin changes. Lengthy discussions of this possibility is helpful in the consultation process to maintain patient confidence in the surgical team in the highly comorbid and possibly already frustrated patient. C-section scars that do not extend laterally often keep the superficial epigastric vessles intact and the lower abdominal skin can be maintained preventing wound complications.

In the absence of abdominoplasty incisions or extended C-section scars, the previous midline incision is utilized. We typically undermine lateral to the anterior axillary line for component separation keeping the lateral intercostal blood supply intact after sacrificing the blood supply from the rectus muscle 
perforators. An intact superficial inferior epigastric system allows venous drainage of the skin flaps and healing occurs readily with minimal skin necrosis.

If there is a question of dead space after the undermining and concern of seroma formation or skin viability, the excess skin can be excised, and a midline horizontal incision can be closed. Drains are used in all cases and abdominal binders are used for six weeks to help prevent seroma formation.

Step 4. What are the comorbidities that the patient presents with and what needs to be optimized prior to surgery?

Both general surgeons and plastic \& reconstructive surgeons need to pay attention to the comorbidities of the patient. Not only does this prevent complications associated with treatment, but it can also facilitate treatment. For abdominal wall reconstruction we use the following rules for patients undergoing abdominal wall reconstruction.

1. Smoking needs to be stopped for 6 weeks before surgery and 6 weeks after surgery. Hyperbaric oxygen consultation is required for patients with a history of smoking.

2. All patients are instructed to begin a diet of $1-2 \mathrm{~g} / \mathrm{kg} /$ day of protein intake, which usually tends to be roughly 80-100 grams of protein per day. We use the rule of 20's: a can of tuna fish is 25 grams, a chicken breast is 25 grams, three eggs are 25 grams, and a protein shake at night is 25 grams, totaling 100 grams. This not only increases protein stores in the patient preoperatively, but also, we have found patients frequently begin to lose weight. Often these same habits persist post-operatively and can theoretically reduce hernia recurrence if patients continue to lose weight.

3. Referral to their primary medical doctor for clearance is paramount. Patients are optimized with respect to $\mathrm{HgA} 1 \mathrm{c}$ and all other medical comorbidities. Anticoagulation is appropriately bridged per hematologists, cardiologists, etc.

Step 5. After the patient is cleared medically for surgery, what are the next steps we need to do to prepare for surgery?

Once the patient has been cleared for surgery, we generally see the patient in our office two weeks prior to surgery for a pre-operative appointment. During the pre-operative appointment, we assess patient's intake of protein over the past several months. Three days prior to surgery the patients consume protein shakes and smoothies and are placed on clear liquids for two days prior to surgery. The patients are given neomycin and erythromycin on the day prior to surgery and on the morning of surgery the patients are instructed to take Emend $40 \mathrm{mg}$ with a sip of water prior to coming to the hospital. We find that this regimen prevents nausea and distension post-operatively and ultimately protects our repair. When feasible the patients receive a spinal anesthetic prior to surgery. The spinal has been helpful for preventing opiate use post-operatively with the intention of facilitating early return of bowel function.

\section{Step 6. What are the intra-operative steps that will take place during surgery?}


In the preoperative holding area the abdominal incisions are re-traced or marked on the abdominal wall as well as an outline of the hernia.[Figure A] The hernia outlines are marked while the patient is supine as well as after being intubated on the operating room table to demarcate area of dissection.[Figure B] Typically the operative procedure commences in the following order.

The skin is incised at the location of the previous incisions and extended to the marked incisions. Dissection with cautery proceeds down to the intact portion of the abdominal wall superior to the abdominal wall hernia. From this point we begin undermining the abdominal flap off of the abdominal wall laterally toward the anterior axillary line. This is done bilaterally. The skin incision is then directed to the abdominal wall inferior to the level of the hernia and then the abdominal wall skin is undermined laterally toward the anterior axillary line, bilaterally. The known superior and inferior dissections around the hernia defect are then joined with the hernia defect itself. At this point, elevating the skin off the hernia sac can be performed safely with good exposure of any bowel or intra-abdominal contents.[Figure C] The skin flaps are then mobilized away from the defect and kept moist with wet lap sponges. [Figure D]

The intra-abdominal component of the procedure commences and the hernia sac is entered and the hernia sac is excised from the abdominal wall.[Figure E] Lysis of adhesions of loops of bowel from the anterior abdominal wall and often occurs in concert with the removal of any abdominal wall mesh or intra-abdominal mesh. The loops of bowel are then examined, and any lysis of adhesions occurs in symptomatic patients.

After this is completed we proceed with component separation of the layers of the rectus sheath for subcostal/lateral hernias and then with release of the external oblique aponeurosis to medialize the rectus muscles.[Figure D, E] This can be done unilaterally or bilaterally. The rectus muscles are approximated with Kocher clamps to determine the give of the abdominal wall and to determine the size of mesh needed for underlay. [Figure F] When possible it is best to have the transfascial suture knots rest on the lateral aspect of excised external oblique aponeurosis or fascia. Knots resting in these lateral core muscles, we believe, can lead to a small amount of muscle necrosis and enzyme leak.

Prolene sutures are then pre placed via $U$ stitch on the Strattice acellular dermal matrices or synthetic mesh [Figure G] and then passed through the anterior abdominal wall in either the intraperitoneal location or in the retro-rectus space depending on the location of the hernia being repaired. [Figure $H$ ] These sutures are then tied and we proceed with the approximation of the rectus muscles to re-create a dynamic abdominal wall. [Figure I] Pre-mapping the location of the transfascial suture placement is helpful. It is important to mark the location of the suture to be passed through the abdominal wall when the midline is closed. [Figure J] Now the abdominal core musculature is restored and there is load sharing between the midline repair and the acellular dermal matrix.[Figure K] JP drains are placed between the acellular dermal matrix and abdominal wall and placement of drains in the subcutaneous space.[Figure L] We then excise any devitalized skin or excess skin to obliterate dead space.[Figure $\mathrm{M}, \mathrm{N}$ ] The incisions are all closed and bacitracin ointment, Xeroform gauze, and an abdominal binder is placed. 
Multiple options exist to repair ventral hernias with posterior and anterior component separations offering different advantages.[21] While the anterior component separation offers the advantage of direct visualization of the external obliques, there is a potential dead space created where fluid can accumulate. Controlling the excess subcutaneous tissue can lead to an abdominal contour improvement which is an advantage of the anterior component release. [Figures O-R\}

\section{Step 7. What are the immediate and longer term post- operative care measures for the patient?}

The patients are admitted to the floor post-operatively. The patients ideally are given spinal for anesthetic and a post-operatively includes IV Tylenol. The patients are encouraged to ambulate post-op day 1. On post-op day two the foley is discontinued and when urinating, Toradol is added for pain control in addition to IV Tylenol. All efforts are made to minimize narcotic use for the resumption of bowel function. Patients are kept npo until passage of flatus. At that point a clear liquid diet is started followed by a regular diet the following day if appropriate. Oral pain meds are initiated when clear liquid diet commences. Patients are allowed to shower on post-op day number 2. JP drains are kept in place until after discharge.

The activity regimen consists of purposeful walking the first week home from the hospital. Patients are told they can walk to the bathroom, walk to eat dinner, and walk to check the mail. The abdominal binder is worn at all times except to shower for six weeks. In the second week the patients are allowed to walk as if they were shopping at the mall. This level of activity continues until the six-week mark. Drains are removed based on output of $<30 \mathrm{cc}$ per drain for three consecutive days and no signs of edema in the tissues.

On the six-week post-operative visit the abdominal binder is discontinued for normal activities of daily living. The patient begins isometric core strengthening with physical therapy at the six-week mark. The patient wears the abdominal binder at physical therapy for two weeks until the eight weeks with the intention of removing the binder for physical therapy at eight weeks. The patients are seen again at 3 months 6 months 12 months and then 2 years. Patients are instructed to come back every year after that, but most people find that their abdominal core health has recovered, and they have moved on with their lives.

\section{Conclusion}

Successful reconstruction of a functional abdominal wall with desirable aesthetic outcomes and minimized recurrence can be gratifying for both physicians and patients. Incision placement is important for adequate exposure and placement of transfascial sutures. Skin breakdown and seroma formation can be minimized by maintaining blood supply and decreasing dead space. Results are variable and are often patient-dependent, but with appropriate systematic planning and experience, general and reconstructive surgeon teams can yield consistent results. 


\section{Abbreviations}

\section{IV-intravenous}

\section{JP- Jackson-Pratt}

\section{Declarations}

Ethics approval and Consent to Participate. This study was submitted to the Providence St. Joseph Health System and Hoag Hospital Memorial Presbyterian IRB for approval. The IRB determined the research as exempt for this project. The data collection for the publication was completed via chart review. Informed consent, obtained from all patients, for photographic consent was obtained in written form for all patients.

Consent for publication. All participants provided written consent for their use of photographs for presentation and publication. Patients are not identified by name in any photographs or text. Patients are aware that in some circumstances, the photographs may make their identity recognizable. In addition, patient identifying information has been removed from images and faces are omitted from photographs.

Availability of data and material: The chart and photographic review material are available from chart review from the office of Brian P. Dickinson, M.D., Inc.

Consent to participate. Photographic consent was obtained from the patients in the chart review for presentation and publication.

Competing interests: The authors have no conflicts of interest and no competing interests.

Funding: There was no funding for the completion of the chart review or preparation of the manuscript.

Authors' contributions: The manuscript work presented has not been published before and it is not under consideration for publication anywhere else. The publication has been approved by all co-authors, as well as by the institute where the work has been carried out.

The authors contributions are as follows:

BPD was responsible for IRB approval, gathering data, photographs, creation of technique and inception of the project. MV was responsible for initial manuscript creation and preparing photographs in presentation. $\mathrm{NVH}$ was responsible for initial manuscript creation and preparing photographs in presentation. AS was responsible for creation of technique. TH was responsible for creation of technique and reviewing/editing manuscript. RS was responsible for creation of surgical technique. LP was responsible for creation of surgical technique and reviewing manuscript. KM was responsible for creation of surgical technique and reviewing manuscript. PW was responsible for creation of surgical technique and reviewing manuscript. SB was responsible for creation of surgical technique and reviewing 
manuscript. AM was responsible for creation of surgical technique and reviewing manuscript. MH was responsible for creation of surgical technique and reviewing manuscript.

Acknowledgements: The authors would like to thank Sundae Zehner for her help in these complex reconstructive cases. Sundae is reliable, dependable, and makes these complex reconstructive cases possible.

\section{Brain P. Dickinson, M.D.}

\section{References}

1. Poulose BK, Adrales GL, Janis JE. Abdominal Core Health-A Needed Field in Surgery. JAMA Surg. 2020;155(3):185-6.

2. Willson JD, Dougherty $C P$, Ireland ML, Davis IM. Core stability and its relationship to lower extremity function and injury. J Am Acad Orthop Surg. 2005;13(5):316-25.

3. Ramirez OM, Ruas E, Dellon AL. "Components separation" method for closure of abdominal-wall defects: an anatomic and clinical study. Plast Reconstr Surg. 1990 Sep;86(3):519-26.

4. Sosin M, Patel KM, Albino FP, Nahabedian MY, Bhanot P. A patient-centered appraisal of outcomes following abdominal wall reconstruction: a systematic review of the current literature. Plast Reconstr Surg. 2014 Feb;133(2):408-18.

5. Kapur SK, Butler CE. Lateral Abdominal Wall Reconstruction. Semin Plast Surg. 2018 Aug;32(3):141146.

6. Fitzgibbons RJ, Forse RA. (2015). Clinical practice. Groin hernias in adults. The New England journal of medicine, $3728,756-63$.

7. Paola Primatest MJ, Goldacre. Inguinal Hernia Repair: Incidence of Elective and Emergency Surgery, Readmission and Mortality. Int J Epidemiol. August 1996;25(4):835-9.

8. Poulose BK, Shelton J, Phillips S, et al. Epidemiology and cost of ventral hernia repair: making the case for hernia research. Hernia. 2012;16:179-83.

9. Beadles CA, Meagher AD, Charles AG. Trends in Emergent Hernia Repair in the United States. JAMA Surg. 2015;150(3):194-200.

10. GBD 2015 Mortality and Causes of Death Collaborators. Global, regional, and national life expectancy, all-cause mortality, and cause-specific mortality for 249 causes of death, 1980-2015: a systematic analysis for the Global Burden of Disease Study 2015. Lancet. 2016;388(10053):1459544.

11. Razavi SA, Desai KA, Hart AM, Thompson PW, Losken A. The Impact of Mesh Reinforcement with Components Separation for Abdominal Wall Reconstruction. Am Surg. 2018 Jun;01(6):959-62. 84(.

12. Butler CE. The role of bioprosthetics in abdominal wall reconstruction Clin Plast Surg 20063302199211. 
13. Jin J, Rosen MJ, Blatnik J, et al. Use of acellular dermal matrix for complicated ventral hernia repair: does technique affect outcomes? J Am Coll Surg. 2007;205(05):654-60.

14. Charles F, Bellows A. Alder \& W Scott Helton. (2006) Abdominal wall reconstruction using biological tissue grafts: present status and future opportunities, Expert Rev Med Devices, 3:5, 657-75.

15. Jin J, Rosen MJ, Blatnik J, et al. Use of acellular dermal matrix for complicated ventral hernia repair: does technique affect outcomes? J Am Coll Surg. 2007;205(5):654-60.

16. Giordano S, Garvey PB, Baumann DP, Liu J, Butler CE. Primary fascial closure with biologic mesh reinforcement results in lesser complication and recurrence rates than bridged biologic mesh repair for abdominal wall reconstruction: A propensity score analysis. Surgery. 2017;161(2):499-508.

17. Holihan JL, Askenasy EP, Greenberg JA, et al. Component Separation vs. Bridged Repair for Large Ventral Hernias: A Multi-Institutional Risk-Adjusted Comparison, Systematic Review, and MetaAnalysis. Surg Infect (Larchmt). 2016;17(1):17-26.

18. Patel KM, Bhanot P. Complications of acellular dermal matrices in abdominal wall reconstruction. Plast Reconstr Surg. 2012;130(5 Suppl 2):216S-24S.

19. Baillie DR, Stawicki SP, Eustance N, Warsaw D, Desai D. Use of human and porcine dermal-derived bioprostheses in complex abdominal wall reconstructions: a literature review and case report. Ostomy Wound Manage. 2007;53(5):30-7.

20. Kissane NA, Itani KM. A decade of ventral incisional hernia repairs with biologic acellular dermal matrix: what have we learned? [published correction appears in Plast Reconstr Surg. 2013 Feb;131(2):427-8]. Plast Reconstr Surg. 2012;130(5 Suppl 2):194S-202S.

21. Hodgkinson JD, Leo CA, Maeda Y, et al. A meta-analysis comparing open anterior component separation with posterior component separation and transversus abdominis release in the repair of midline ventral hernias. Hernia. 2018;22(4):617-26.

\section{Figures}




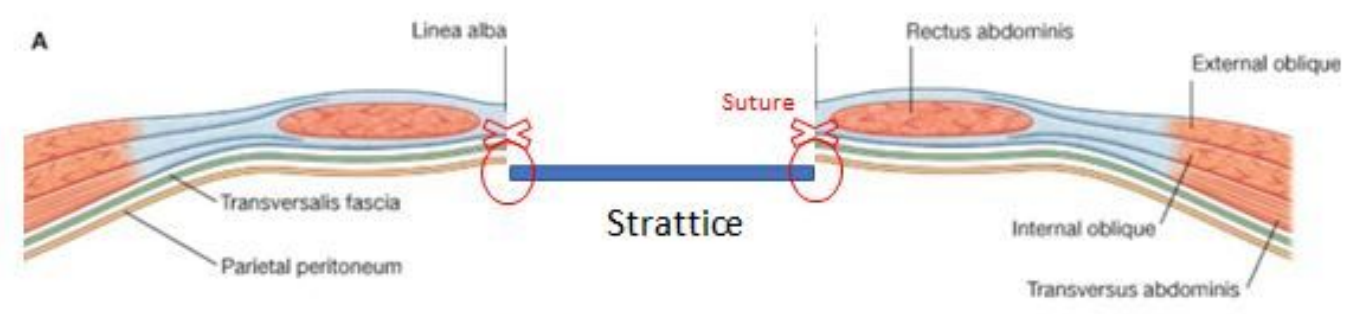

Load Bearing Hernia Repair

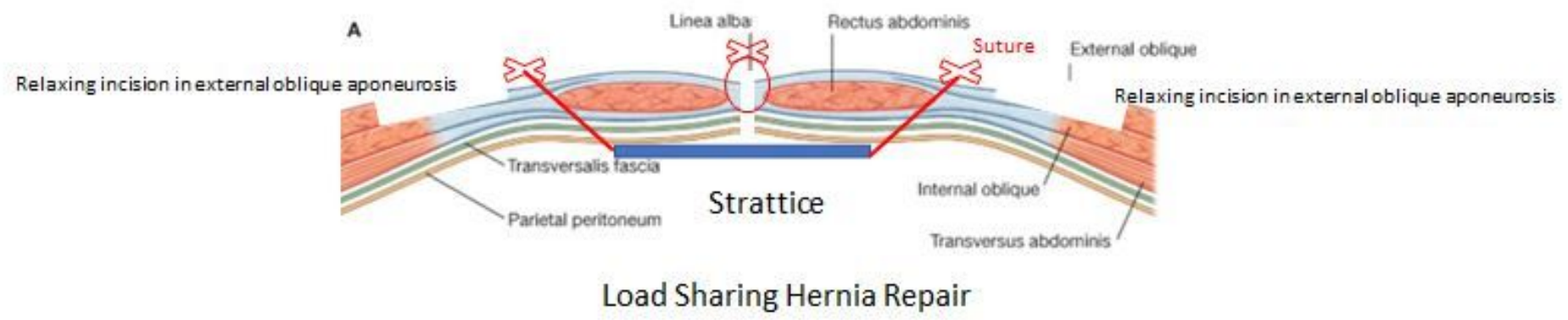

\section{Figure 1}

Diagram 1

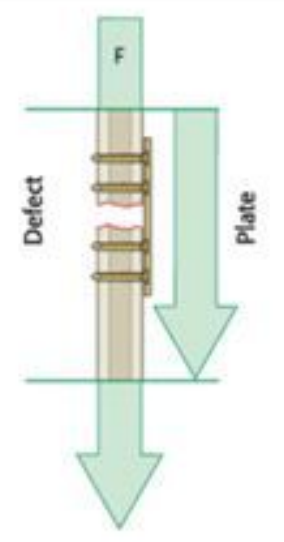

Load bearing

In load-bearing fixation the plate assumes $100 \%$ of the functional loads.

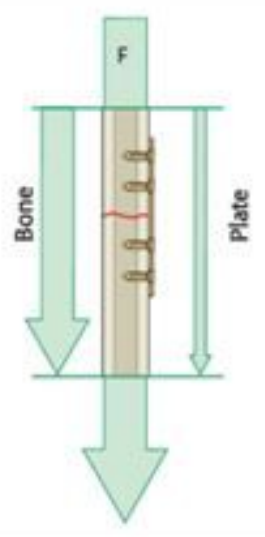

Ideal load-sharing situation

Ideal load-sharing situation where the bone assumes most of the functional loads. 
Figure 2

Diagram 2

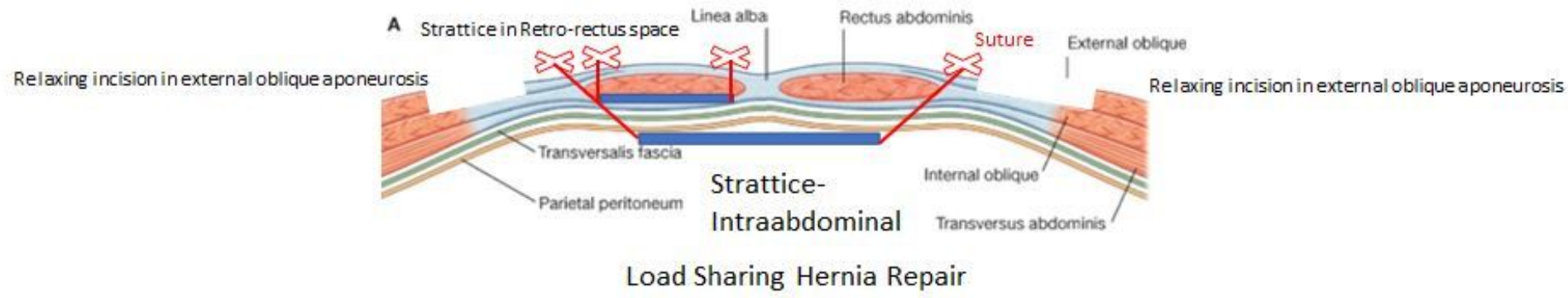

Figure 3

Diagram 3

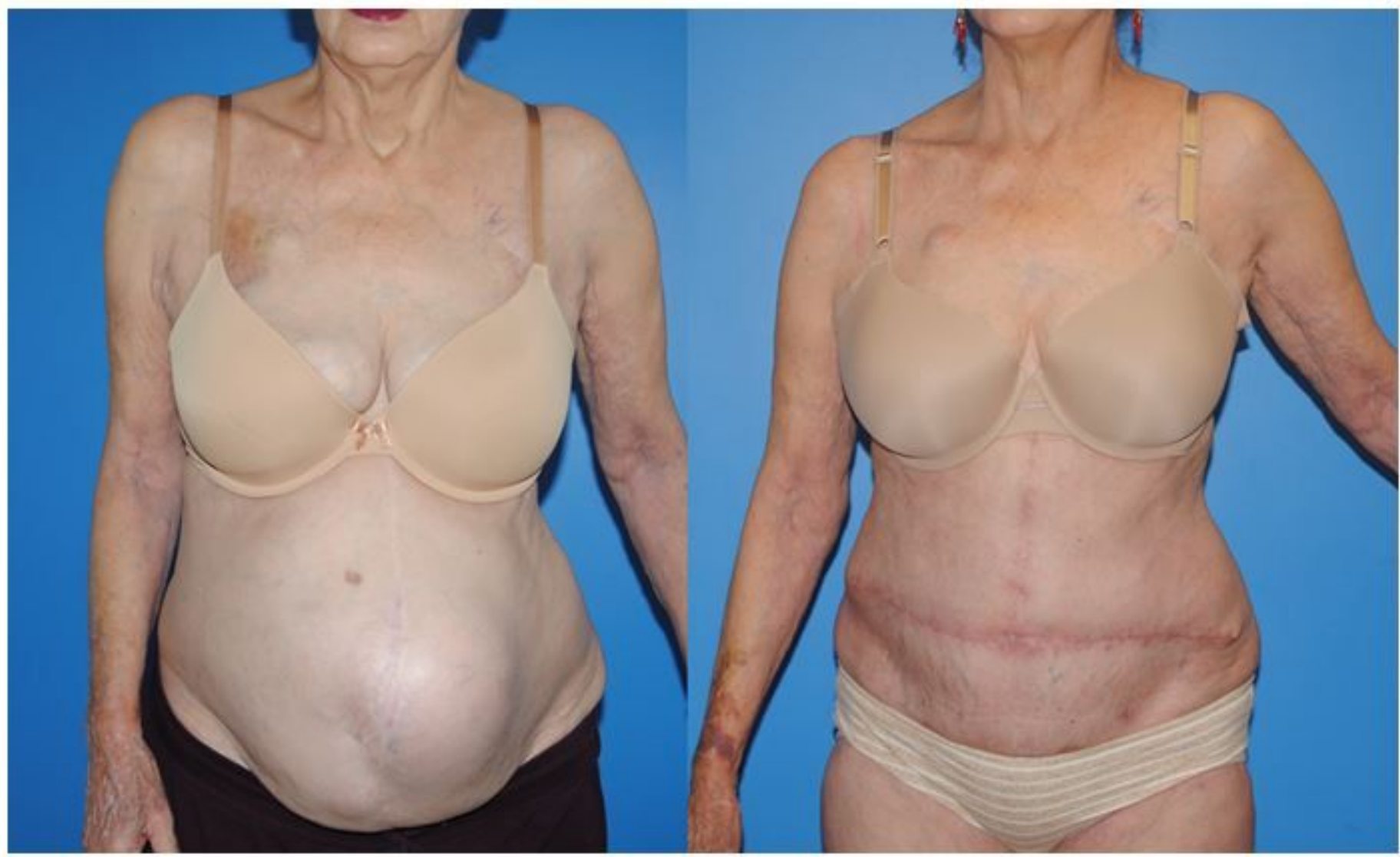

Figure 4

Abdominal wall reconstruction of a midline recurrent ventral hernia. Previous abdominal operations included vertical and lower transverse abdominal scars. The external oblique aponeurosis was released bilaterally with underlay Strattice placement and re-approximation of the rectus abdominis muscles. 


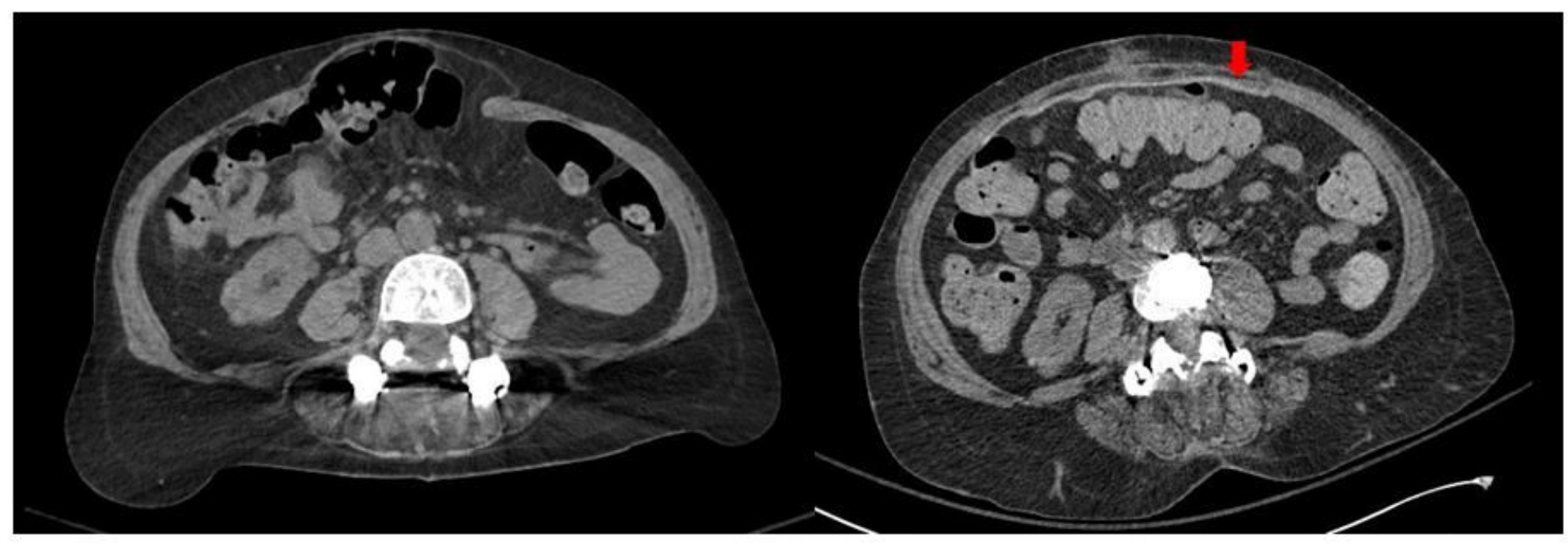

\section{Figure 5}

Pre- and post-operative CT scan showing reapproximation of the rectus abdominis muscles and continuity of the lateral core musculature after external oblique release. The Strattice underlay mesh is visible and in position intraperitoneally. A functional abdominal wall is restored.

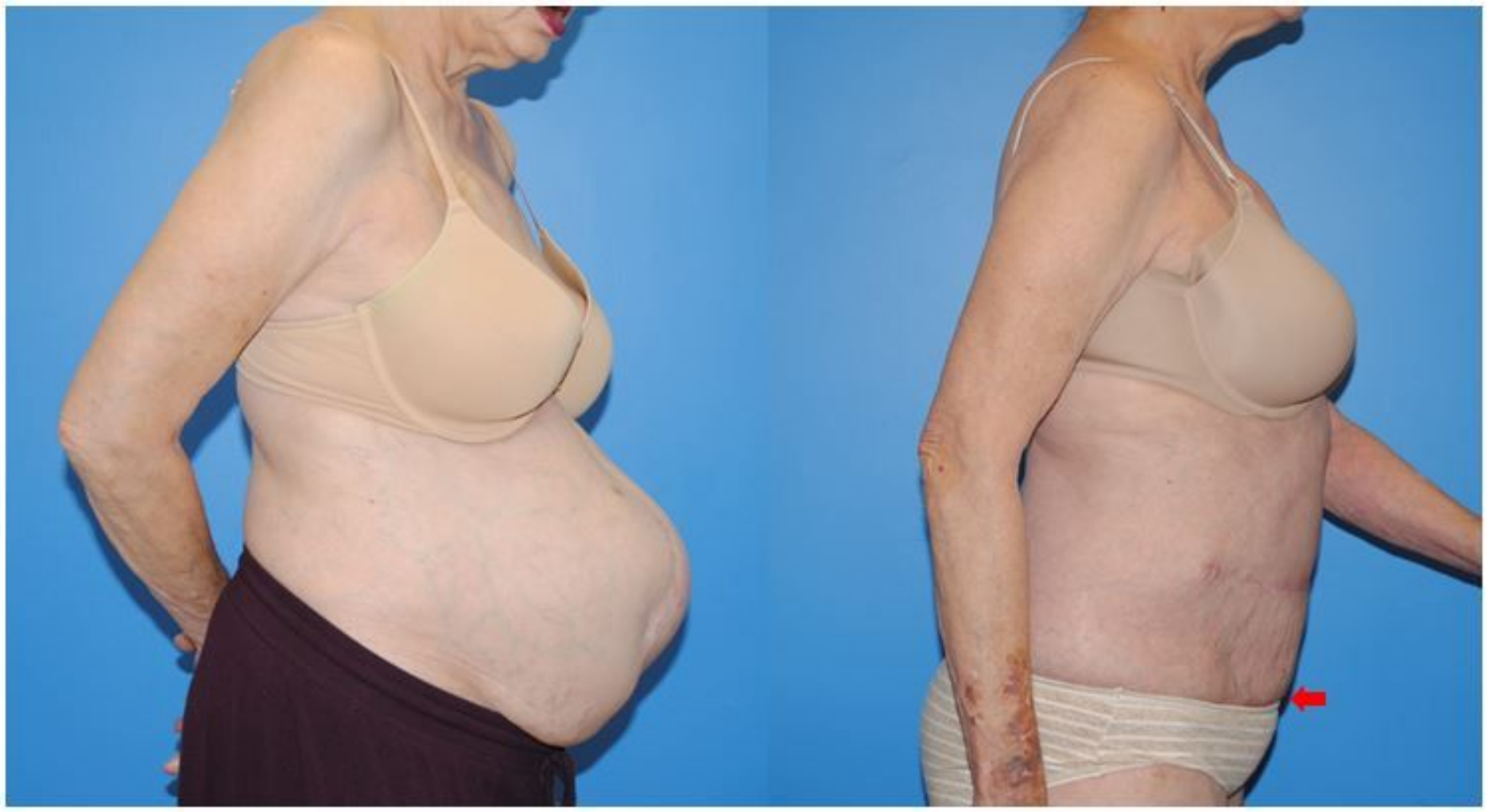

\section{Figure 6}

Despite the previous lower abdominal incision, the lower abdominal skin retained its viability from the superficial circumflex epigastric vessels. The undermined skin and skin over the hernia sac was removed to eliminate dead space. Patients tolerate the high scar well given the improvement in the abdominal contour and absence of bulge. 


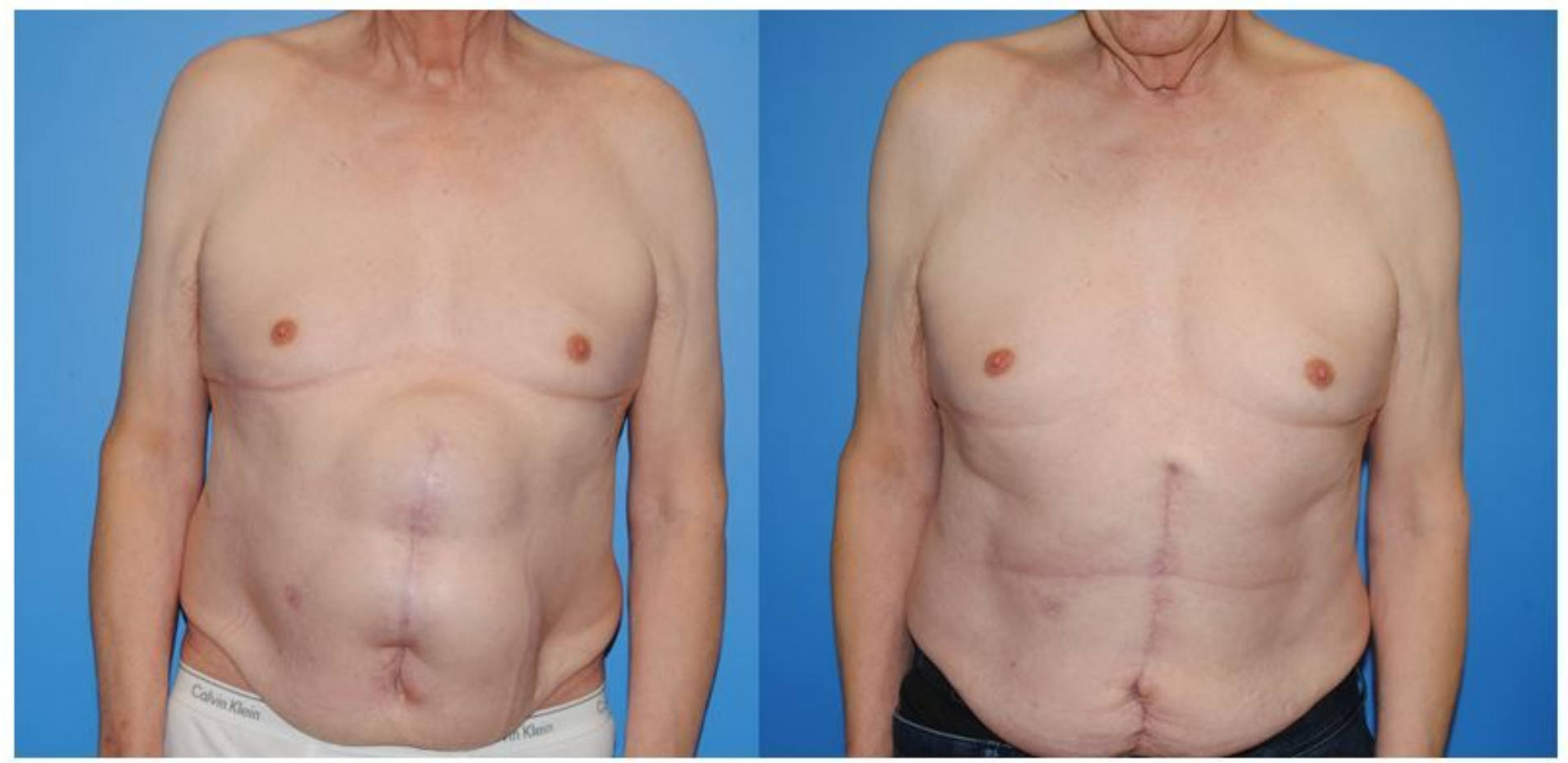

\section{Figure 7}

Large ventral incisional hernia of the abdominal wall. The midline incision was extended. The umbilicus was maintained on the right lateral abdominal skin flap. Undermining was extended to the anterior axillary line and the external oblique aponeurosis released. The Strattice was placed as an underlay. The umbilical stalk was then attached back to the abdominal wall.

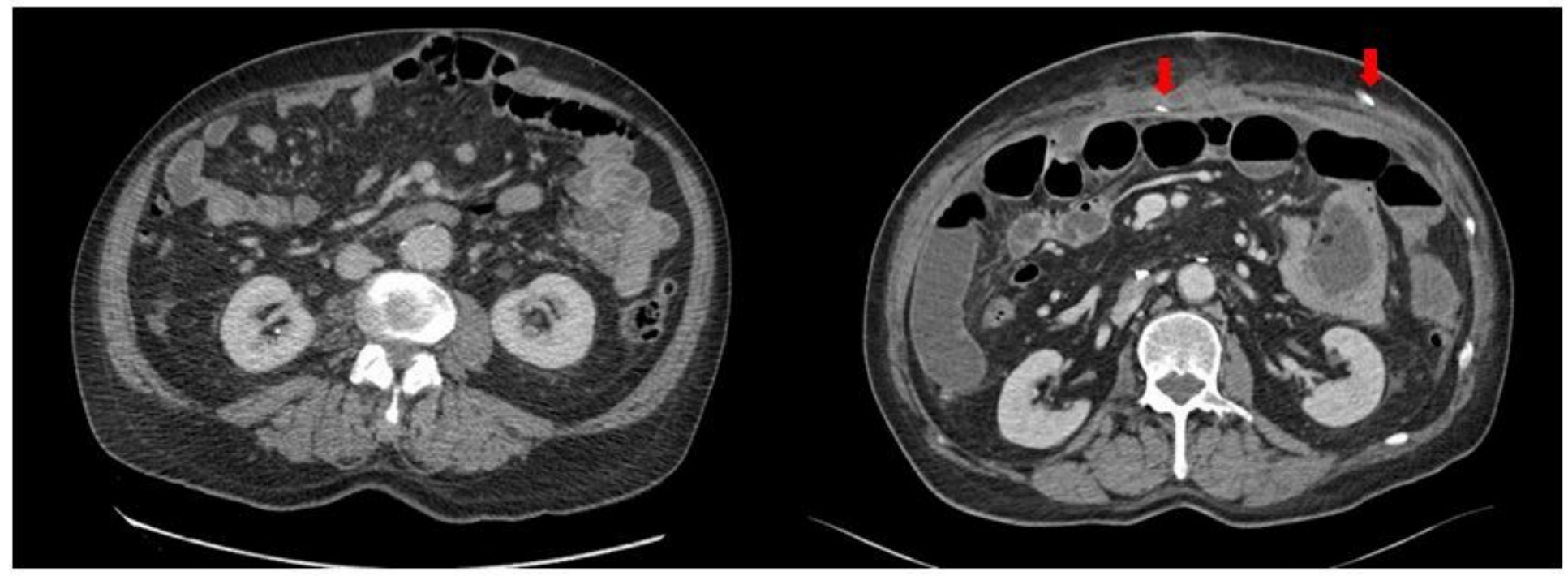

\section{Figure 8}

Pre- and post-op CT scan depicting "Load sharing" hernia repair with re-approximation of the rectus muscles and the Strattice underlay within the peritoneum. Placement of a JP drain between the Strattice 
and the anterior abdominal wall and in subcutaneous positions are helpful to prevent fluid collections and seroma formation.

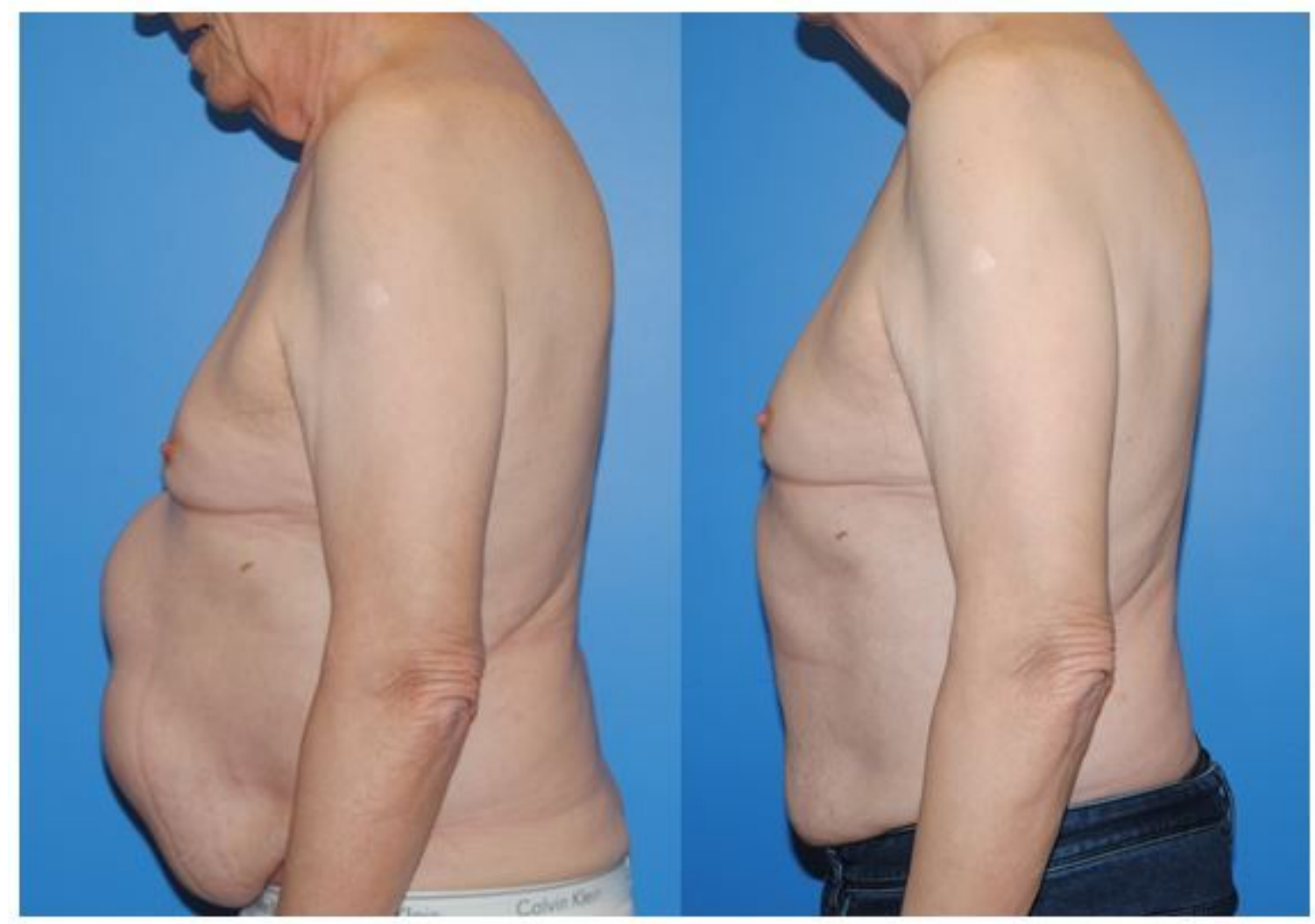

\section{Figure 9}

Repairing the abdominal wall musculature helps to prevent hernia recurrence. Minimal skin was excised in this case as the umbilicus was reattached to the abdominal wall from the right lateral based flap. Restoring the musculature recreates a dynamic abdominal wall with the Strattice "sharing" the load. Lower back pain often improves in these scenarios when abdominal core health is restored. 


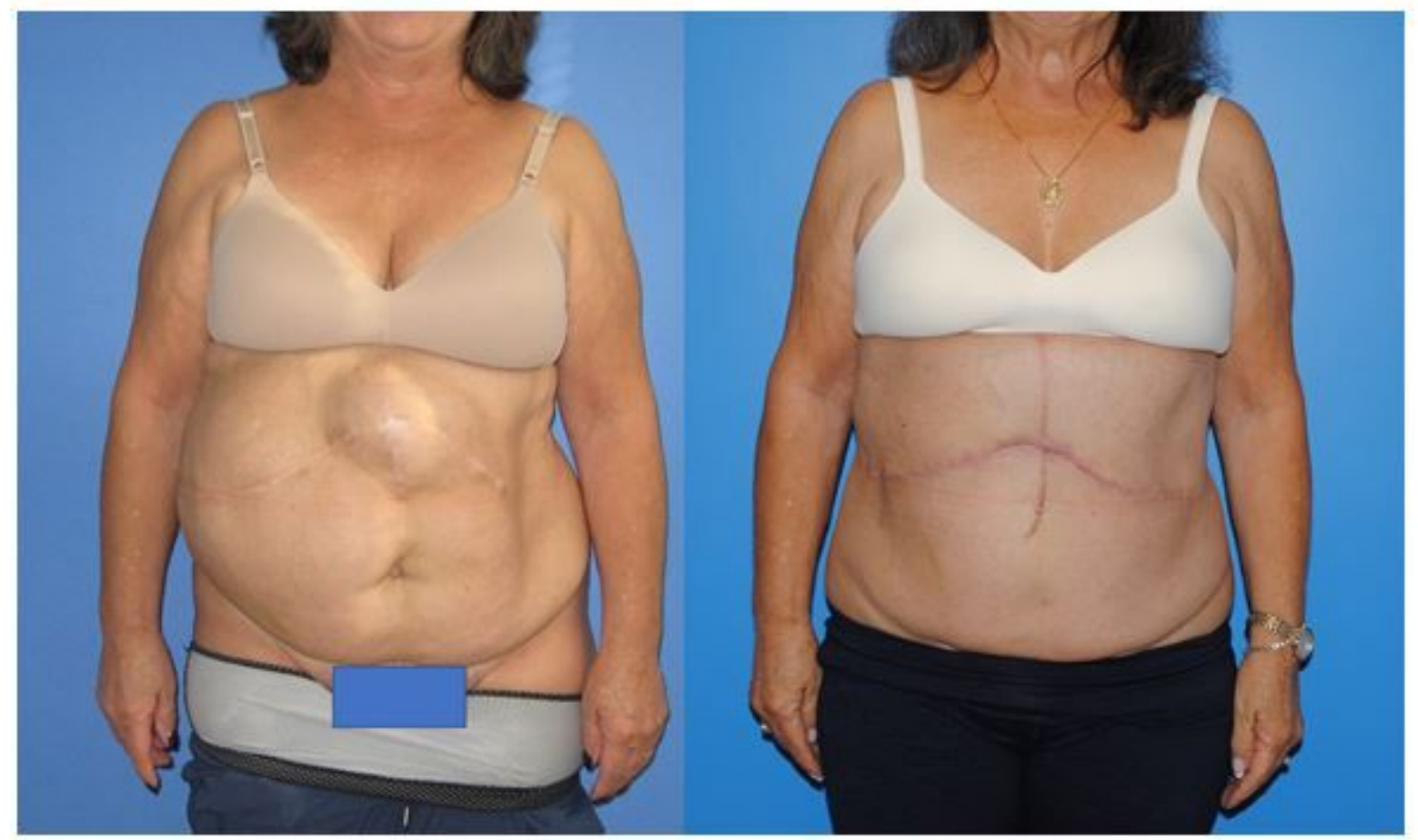

\section{Figure 10}

Recurrent incisional ventral hernia abdominal wall status post right subcostal incision. When repairing the subcostal hernia and midline hernia, the subcostal hernia rectus sheath components are released. Strattice is placed in the retro rectus space in line with the rectus muscle. The order of repair is 1. posterior sheath, 2. Strattice underlay, 3. rectus muscle repair, 4. anterior sheath repair. Undermined skin above hernia sac and to external oblique release is excised to avoid dead space.

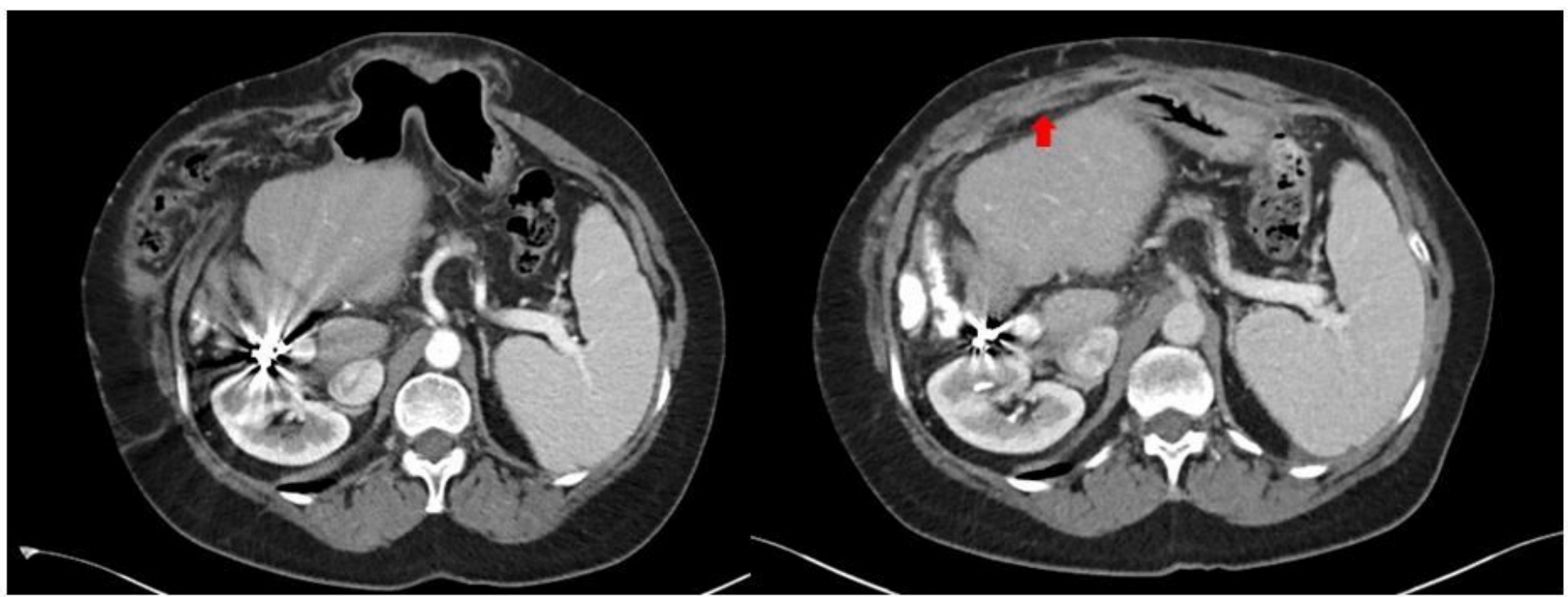

\section{Figure 11}

Pre- and post-operative CT-scan showing repair. When repairing lateral and midline hernias, line up the midline first. Then repair the lateral hernia first and work toward the middle. The common error is utilizing 
all tissue for the lateral repair and then there is limited tissue to mobilize medially and the midline will need to be bridged as a load bearing repair and will not have any functional muscle. This would increase the risk of midline recurrence. Strattice is placed in the retrorectus space.

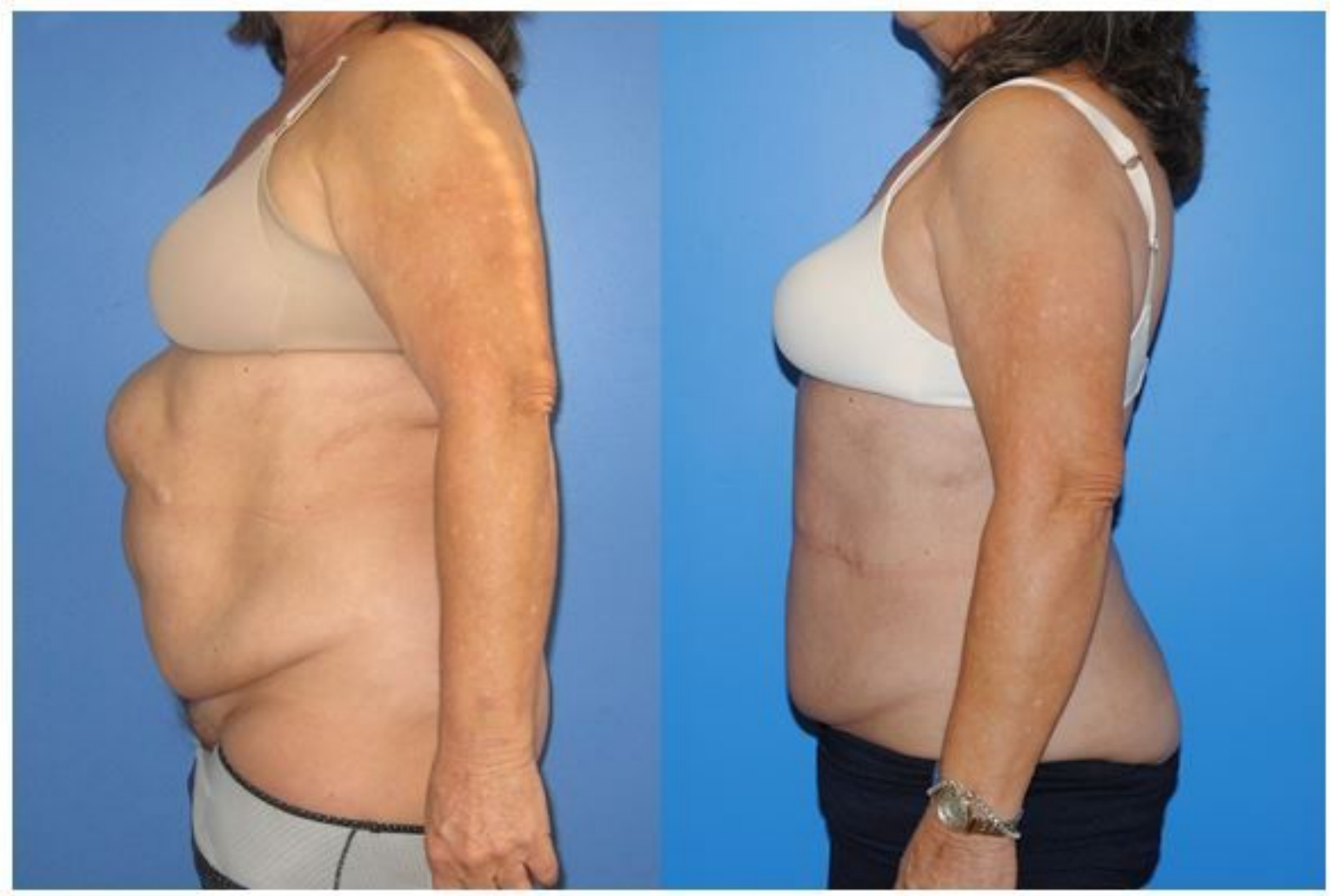

\section{Figure 12}

The order of repair of the rectus sheath is important. The first step is to line up the medial margins of rectus sheath first. Beginning laterally and moving medially will prevent proper alignment of the medial aspect of the repaired sheath. The repaired sheath is then sutured to the contralateral rectus muscle to recreate a dynamic abdominal wall. Successful repair with a load sharing Strattice underlay restores a dynamic abdominal wall. 


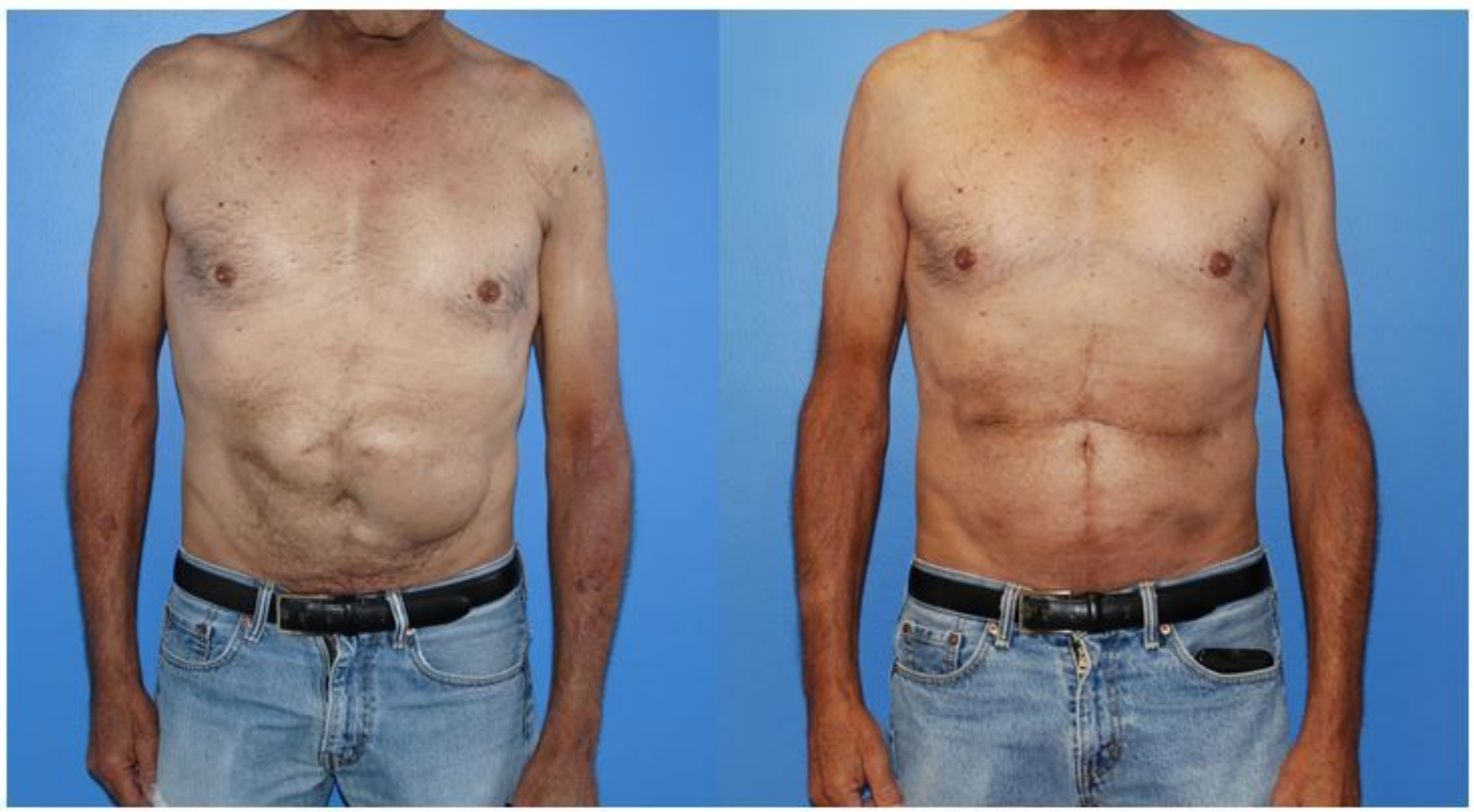

\section{Figure 13}

The medial segments of the repaired left posterior sheath were repaired. The right rectus sheath repaired in a similar fashion on its most medial aspect. The repaired posterior sheaths were then sutured to each other after the right external oblique release. The functional rectus muscles restores a dynamic abdominal wall.

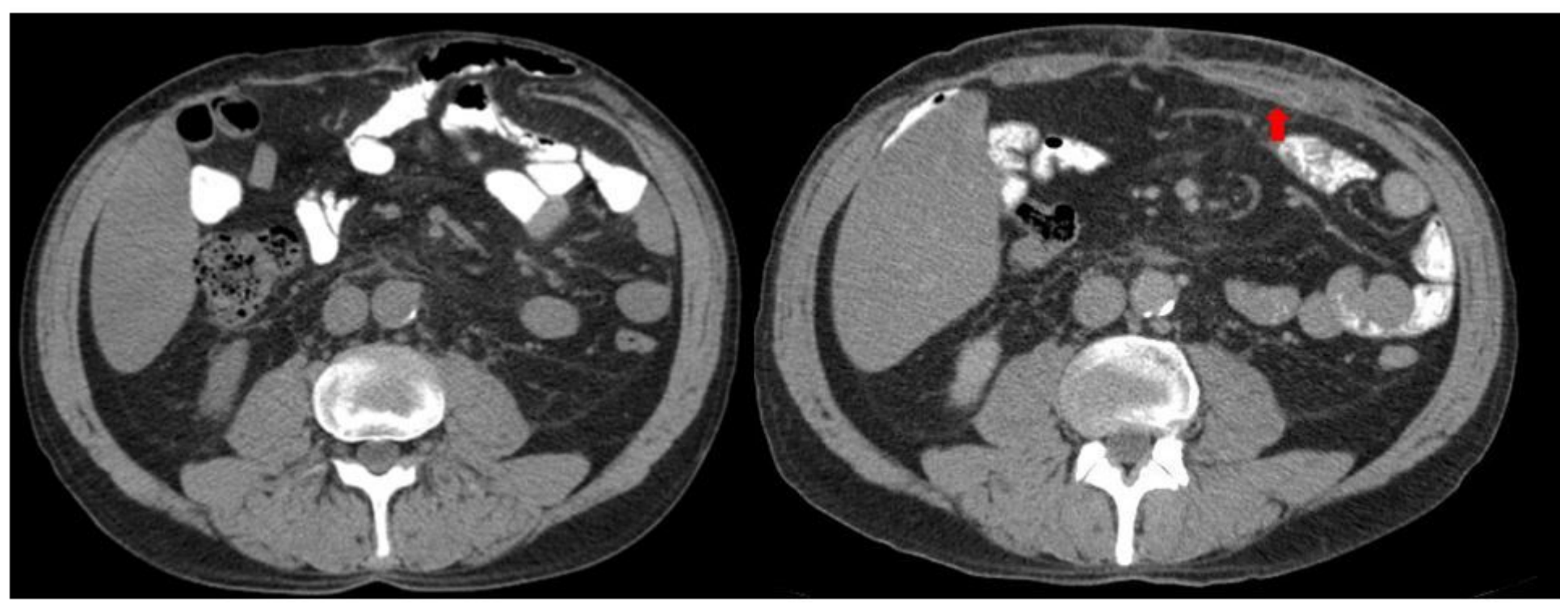

Figure 14 
Atrophic rectus muscles after subcostal incisions are still innervated given the segmental innervation of this muscle. Re-approximation of the muscle can still help to maintain a functional abdominal wall. Strattice is placed in the retrorectus space.

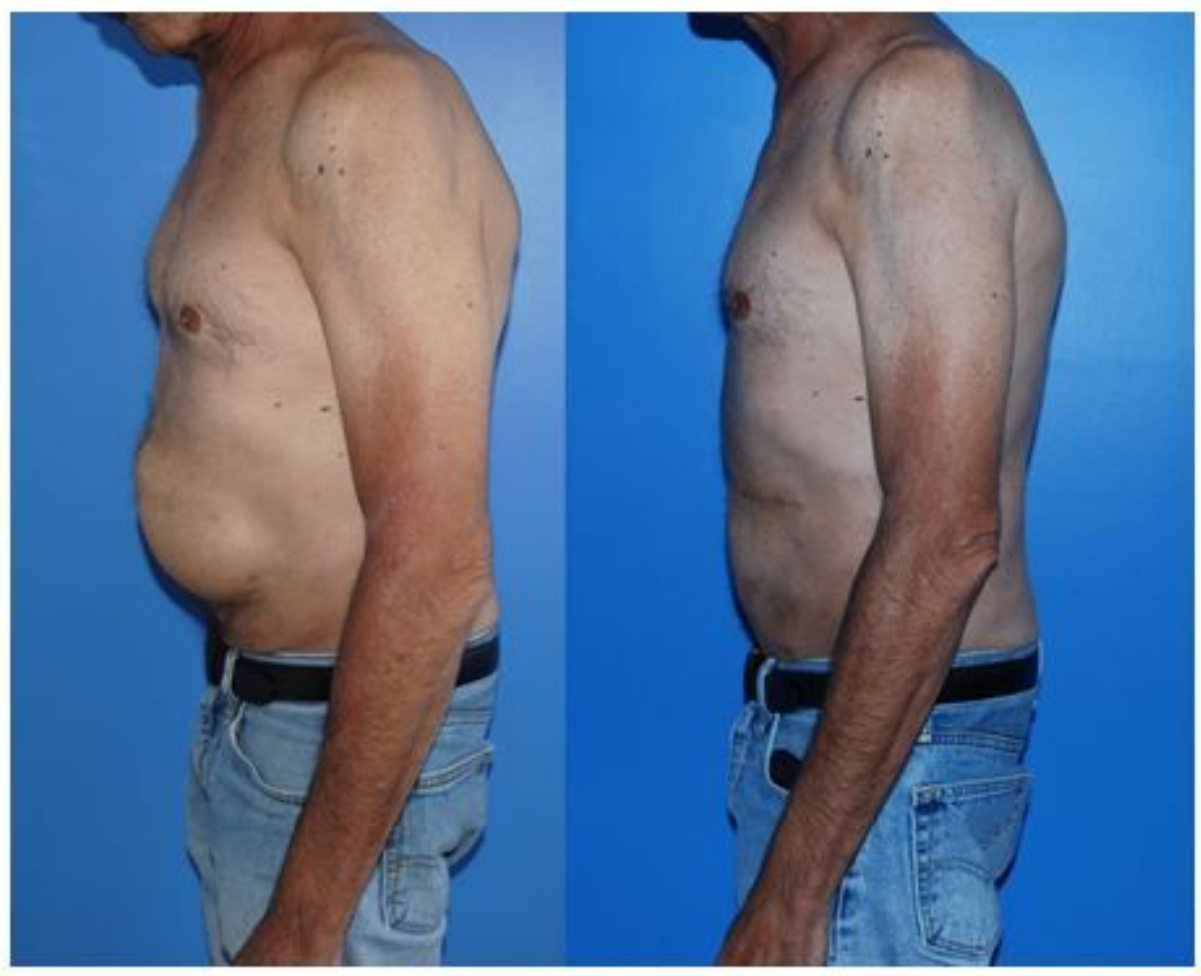

\section{Figure 15}

Repair of the subcostal hernia was completed with component separation of the rectus sheath. The posterior sheath was repaired and Strattice was placed in the rectro-rectus space longitudinally posterior to the rectus muscle. The rectus muscle was approximated. Given the segmental innervation of the rectus muscle, the muscle was functional albeit atrophied. 


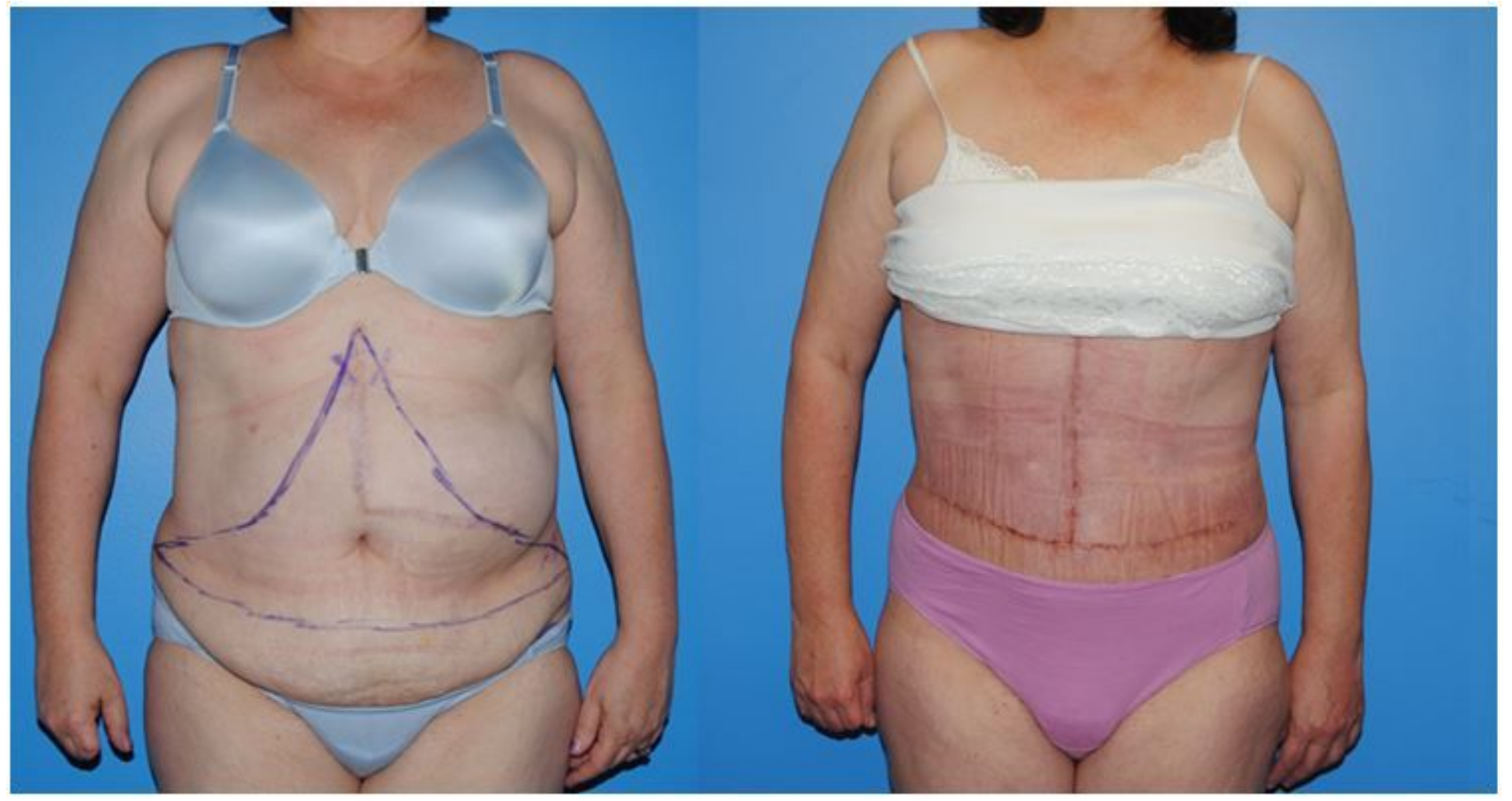

\section{Figure 16}

Careful control of the diabetic Hemoglobin A1C is important prior to surgery. The initiation of a high protein diet of 80-100 mg of protein a day not only increases albumin levels but also assists in glucose control. When blood supply to the inferior abdominal skin flap can be preserved, wound complications can be minimized or more easily addressed.

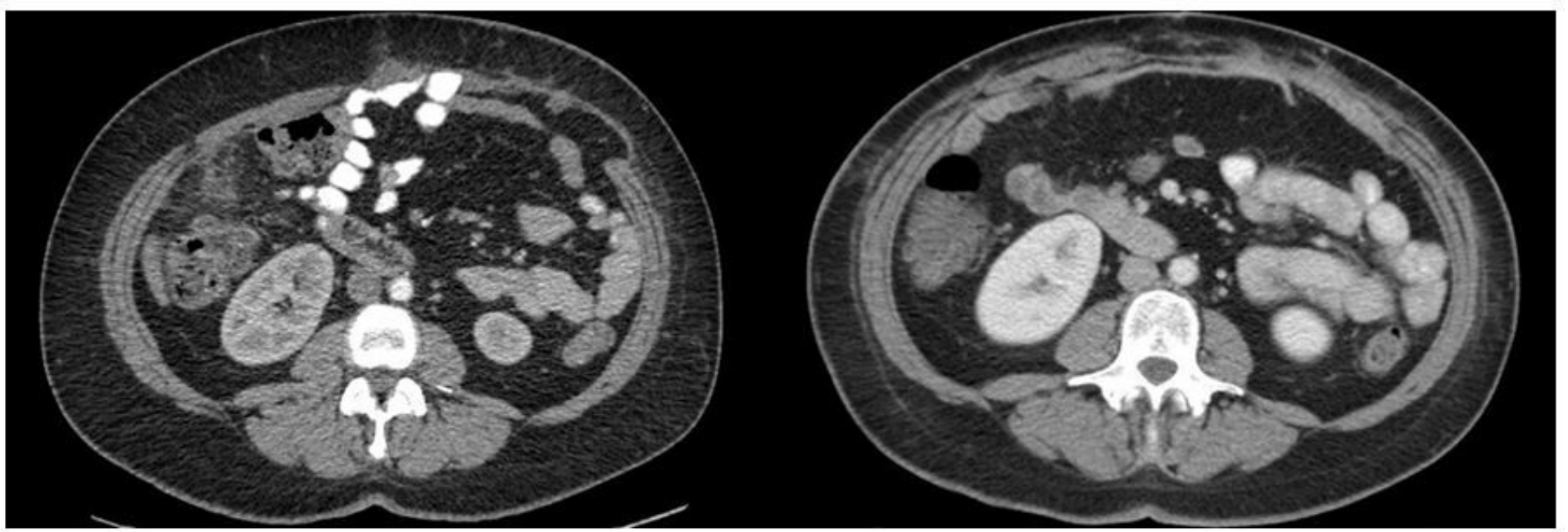

\section{Figure 17}

The subcostal hernia often has a midline hernia in conjunction. It is important when repairing these hernias to make sure that the midline is lined up first. If one starts repairing laterally and moves more medially, the midline does not line up as the abdominal wall is "stolen" laterally. This leaves less tissue 
medially for repair and a non-functional bridge of mesh is required. Line up the midline first with a number one prolene stitch first and then work from lateral to medial.

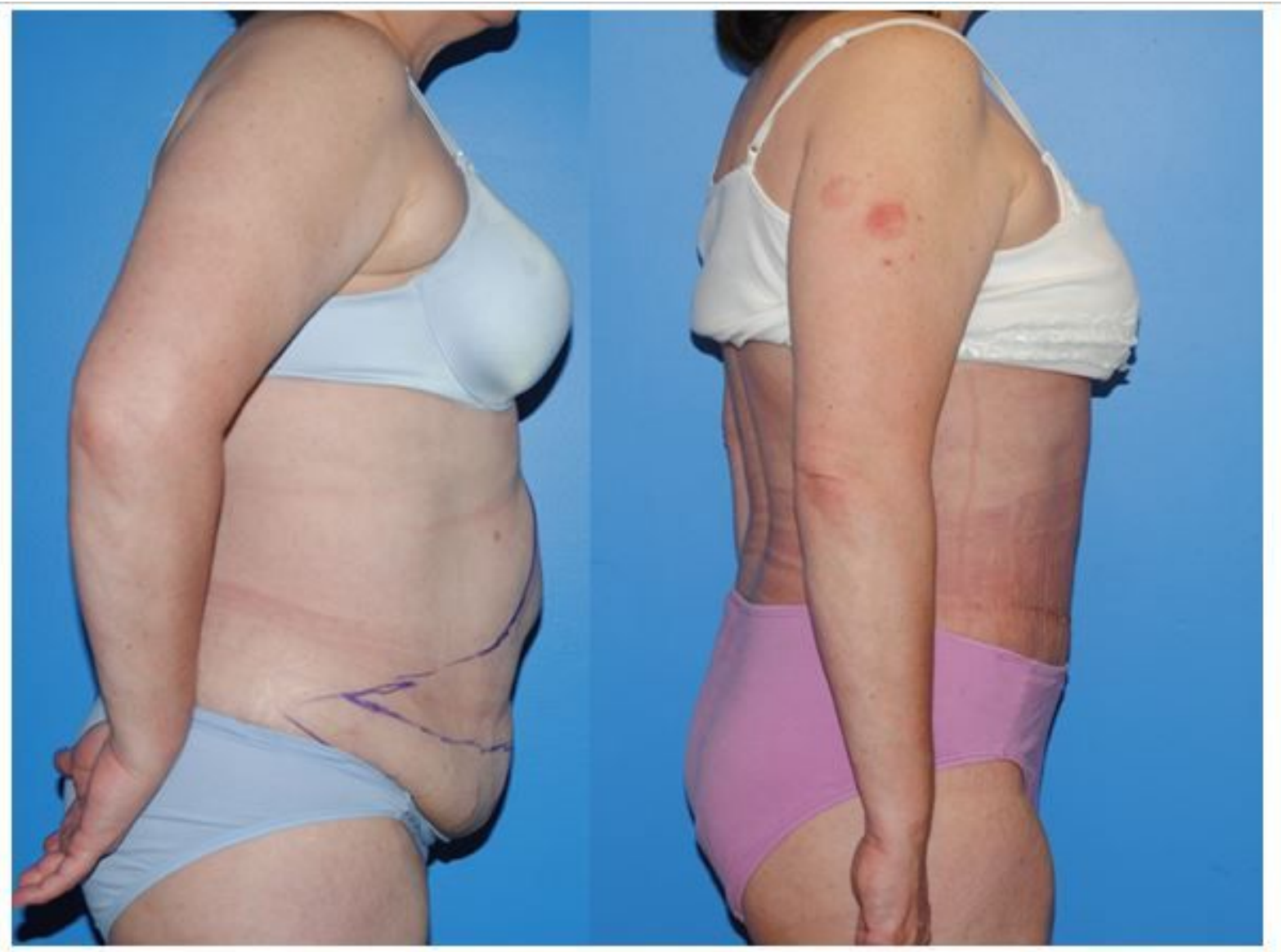

\section{Figure 18}

The superficial inferior epigastric vessels and superficial circumflex epigastrics are important to maintain the venous drainage of the lower abdominal skin flap. Skin necrosis is often a venous issue related to venous congestion of superiorly based flaps. Keeping an inferior based flap can mitigate this problem. 


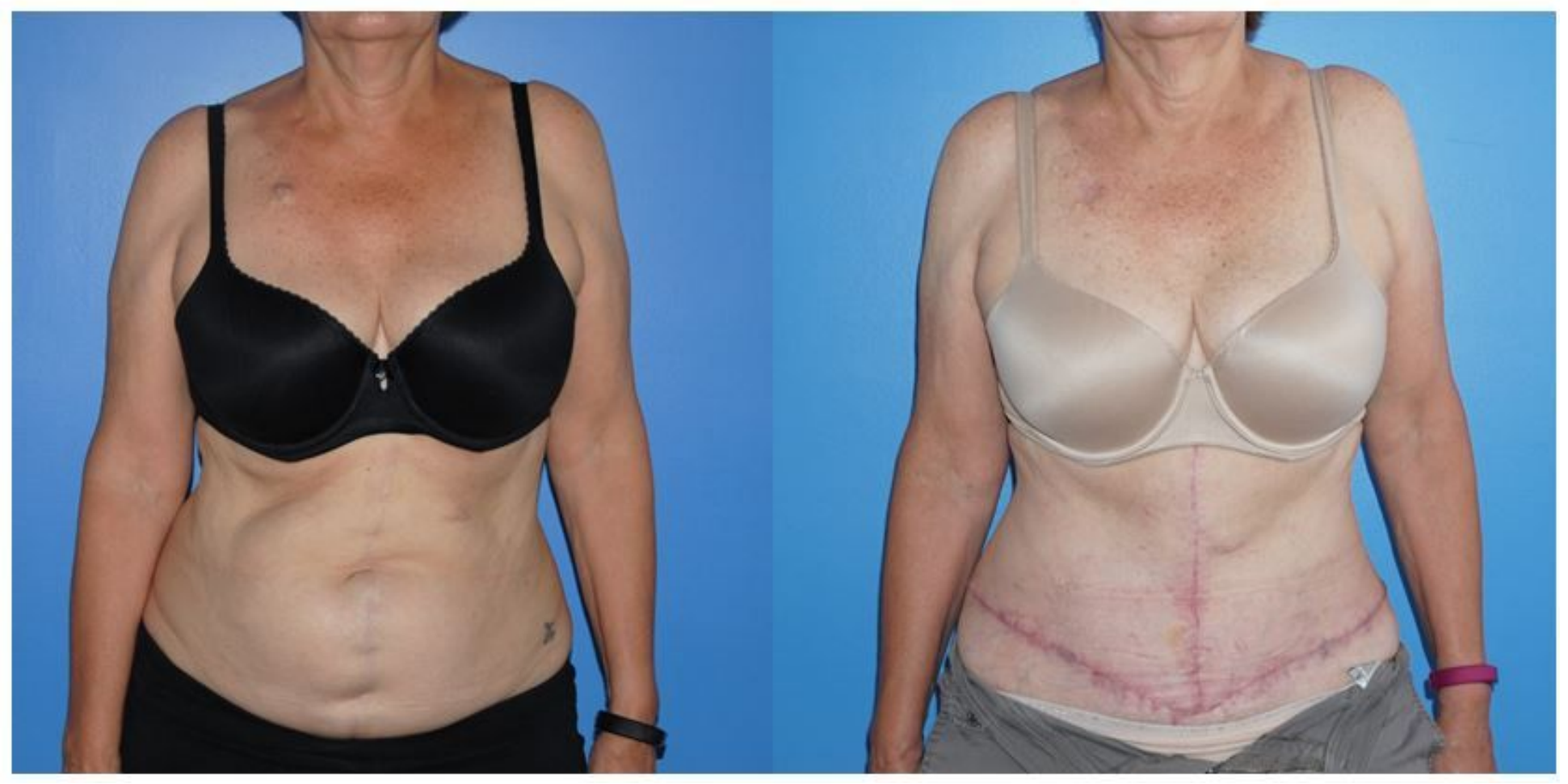

\section{Figure 19}

Ventral incisional hernia with previous midline and lower abdominal scars. The previous midline incision and lower abdominal scars were joined resulting in an inverted T. The patient did not want mesh placed, synthetic or biologic and the repair was completed with anterior component separation only. It was decided to remove the umbilicus pre-operatively.

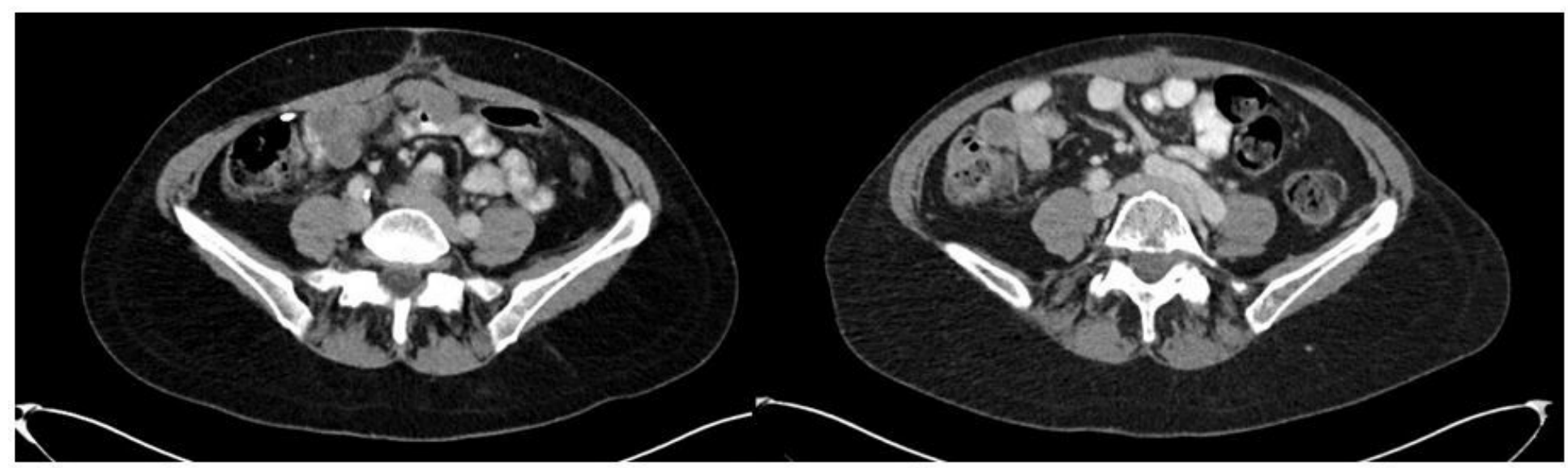

\section{Figure 20}

Ventral incisional hernia with previous midline and lower abdominal scars. In the patient with a low BMI who is not extremely active, hernias can be successfully repaired with component separation and without additional synthetic or biologic mesh. This approach without using mesh to "load share" should be used cautiously in patients with a high BMI and in patients who are physically active. 


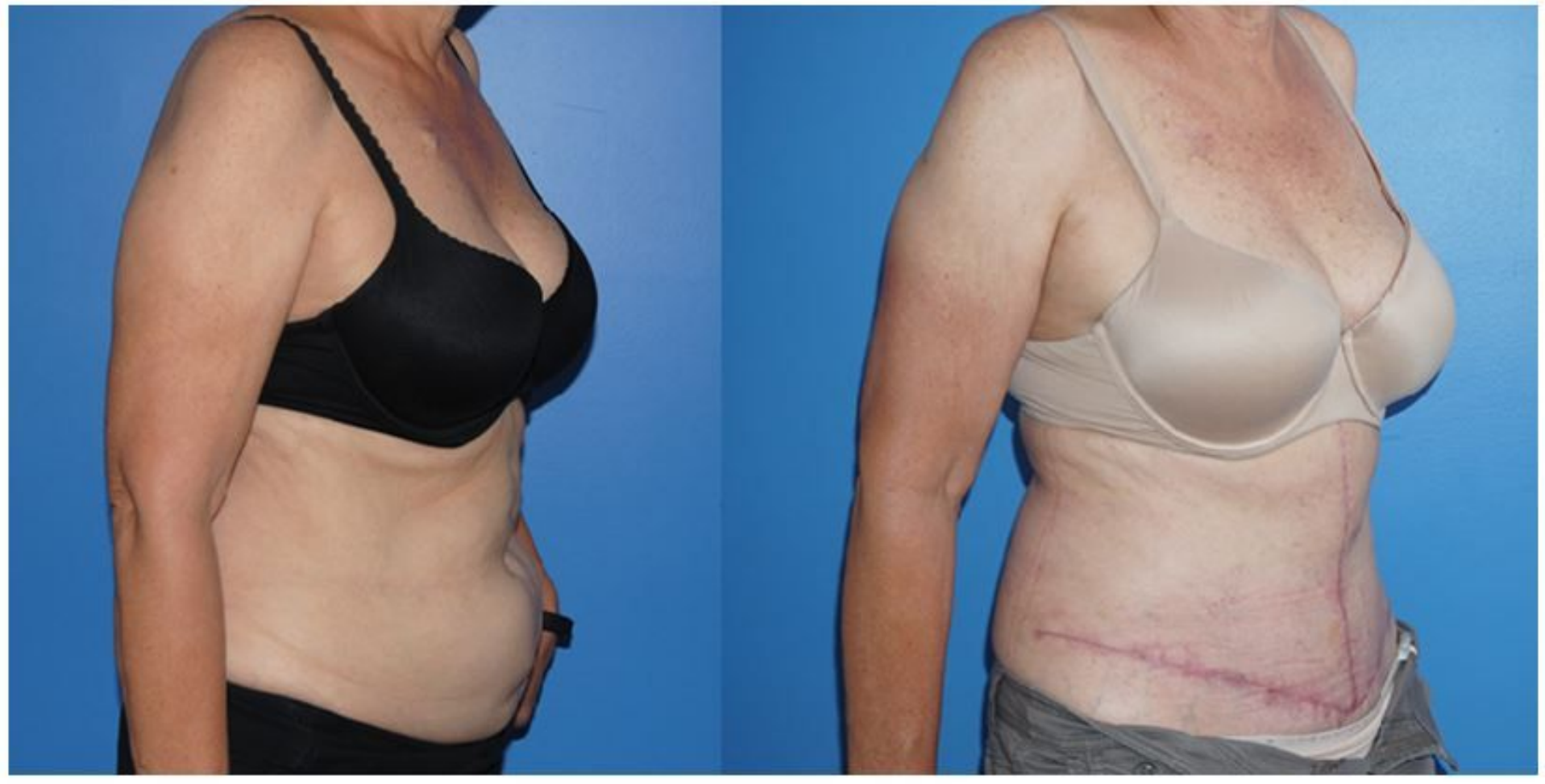

\section{Figure 21}

The previous midline incision and lower abdominal scars were joined resulting in an inverted T. This skin pattern is well tolerated in thin patients with normal BMI. In larger patients the venous return of the distal end of the flap is impaired and large amounts of skin necrosis can occur.

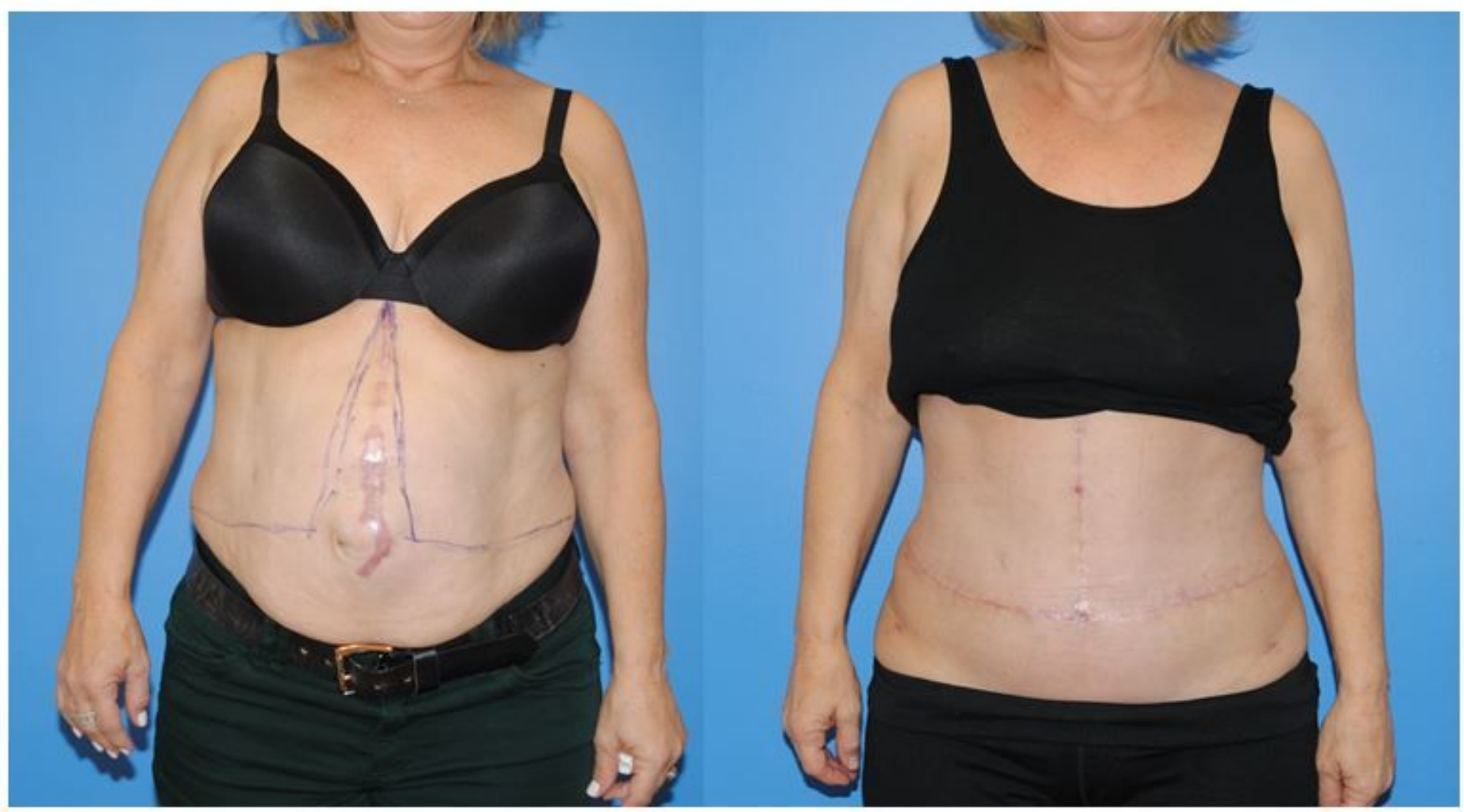


Figure 22

The previous midline incision was maintained to just below the umbilicus. The superficial epigastrics and external intercostals kept the lower abdominal skin viable. We performed minimal undermining of the lower abdominal skin. Given the thin atrophic skin around the umbilicus, it was decided preoperatively to resect the umbilicus.

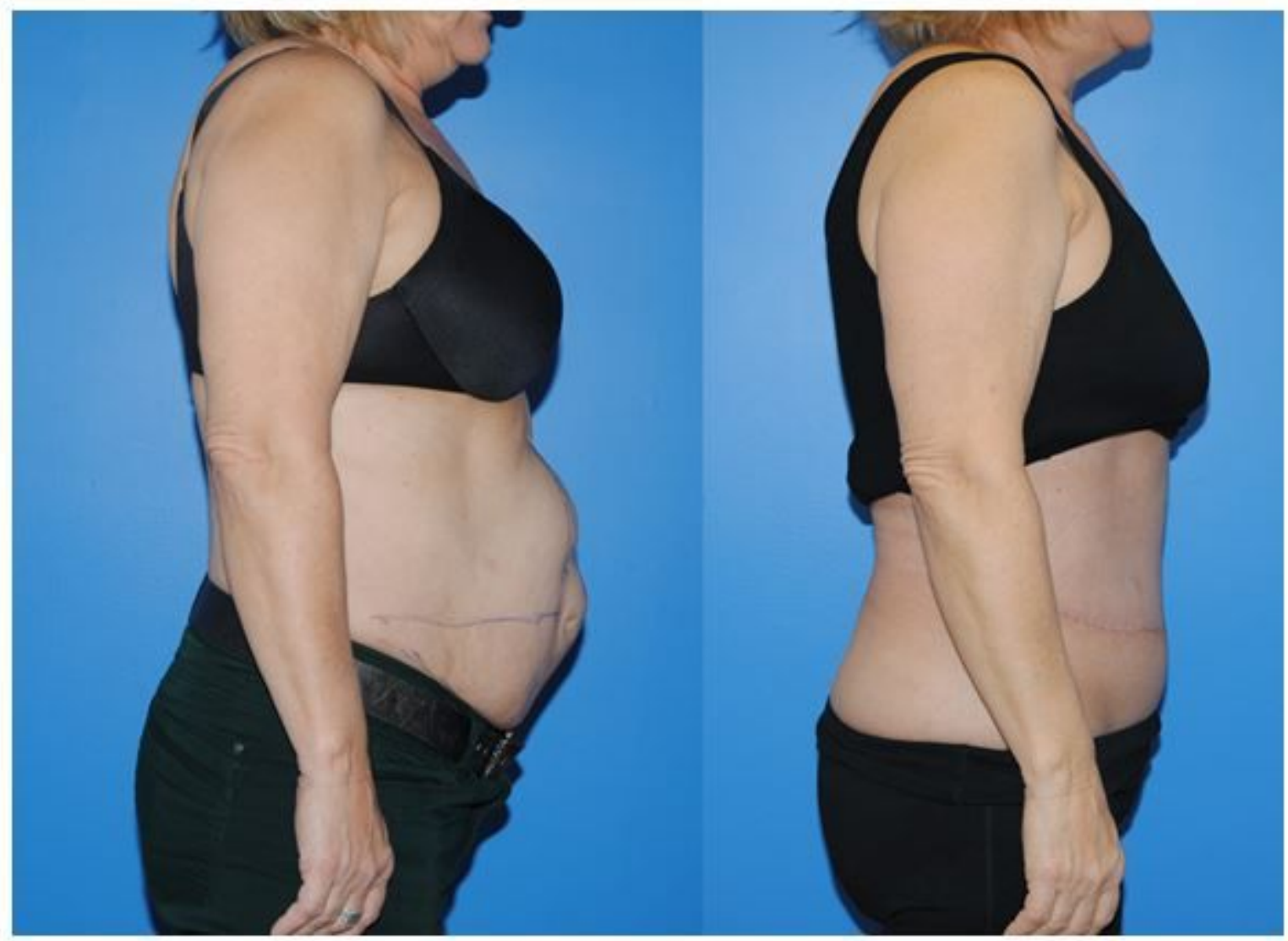

\section{Figure 23}

The previous midline incision was maintained to just below the umbilicus. The superficial epigastrics and external intercostals kept the lower abdominal skin viable. We performed minimal undermining of the lower abdominal skin. 


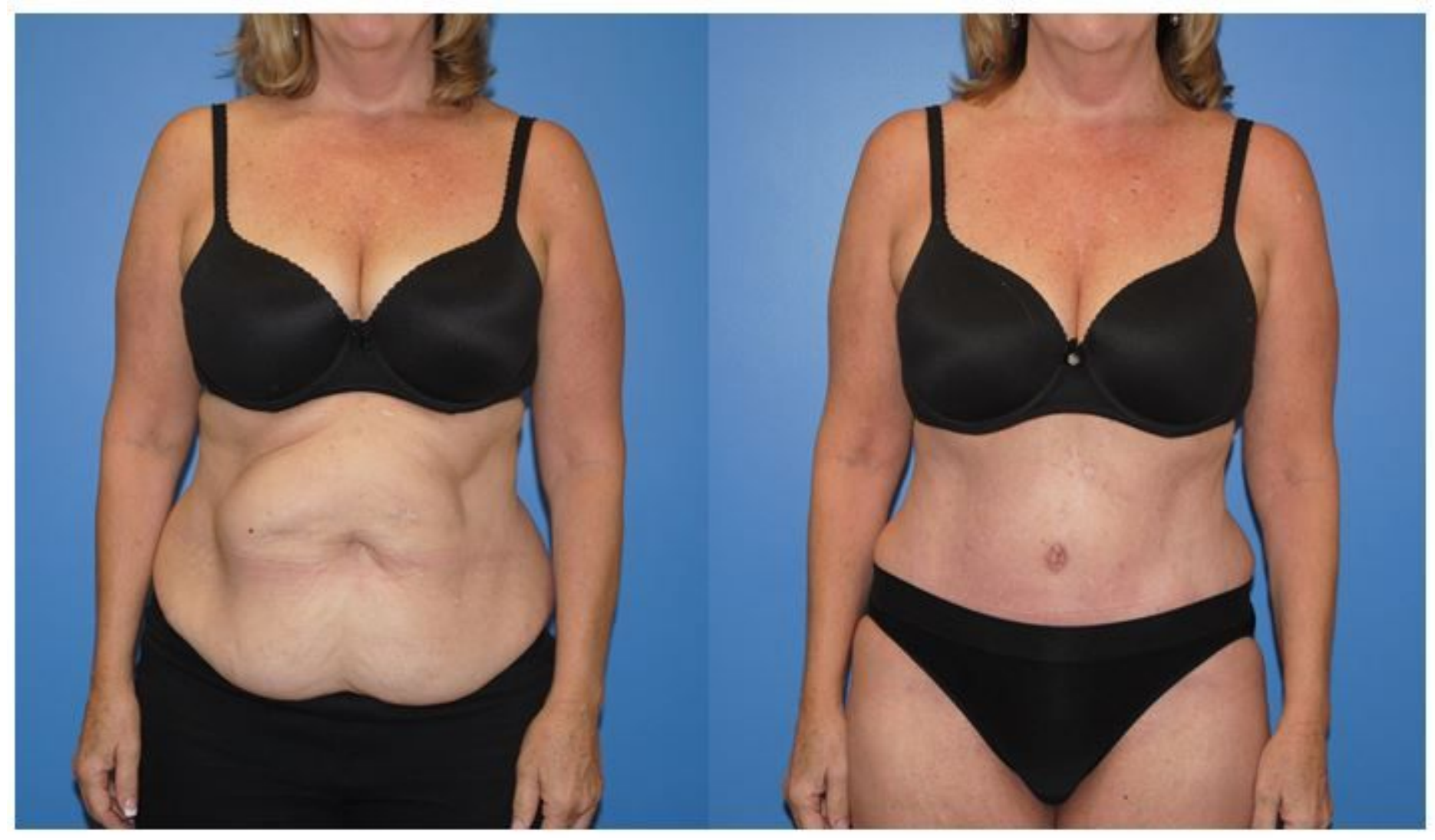

\section{Figure 24}

Recurrent ventral incisional hernia. The previous lower abdominal incision was extended laterally. The previous scar was used and the skin was elevated superior to the hernia to the costal margin. Underlay repair of the hernia was completed as well as re-approximation of the rectus muscle, sharing the load of the mesh and restoring a dynamic abdominal wall. 


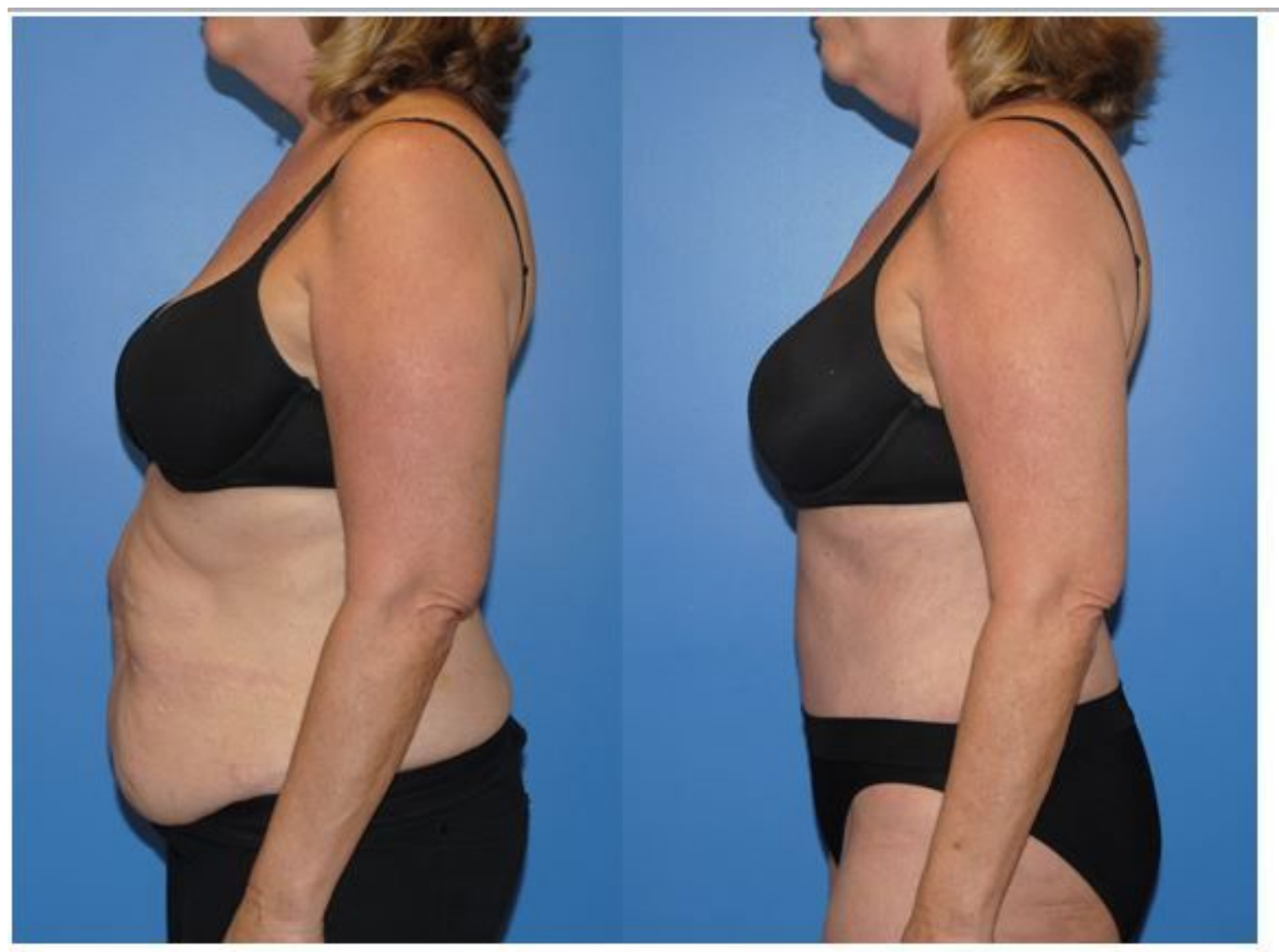

\section{Figure 25}

Recurrent ventral incisional hernia. The previous lower abdominal scar extended laterally. The previous scar was used and the skin was elevated superior to the hernia to the costal margin. Underlay repair of the hernia was completed as well as re-approximation of the rectus muscle, sharing the load of the mesh and restoring a dynamic abdominal wall. 


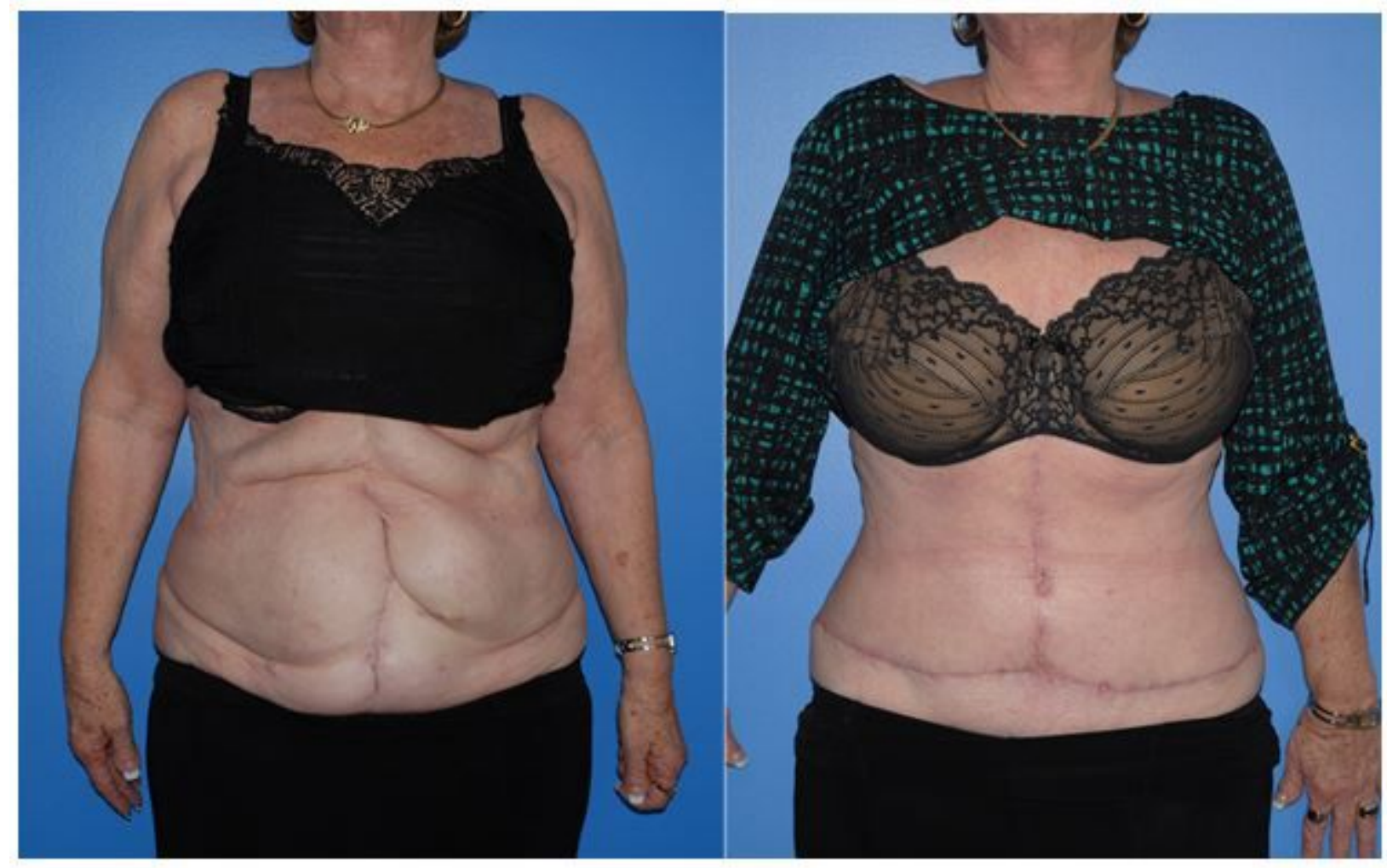

\section{Figure 26}

Ventral hernia with previous midline and lower abdominal scars. Because of the redundant skin superiorly and tethered scars a lower abdominal incision was used which was joined with the previous midline incision resulting in an inverted T. These patients are counseled about the viability of the umbilical stalk following hernia repair as it is often devascularized after underlay mesh placement. 


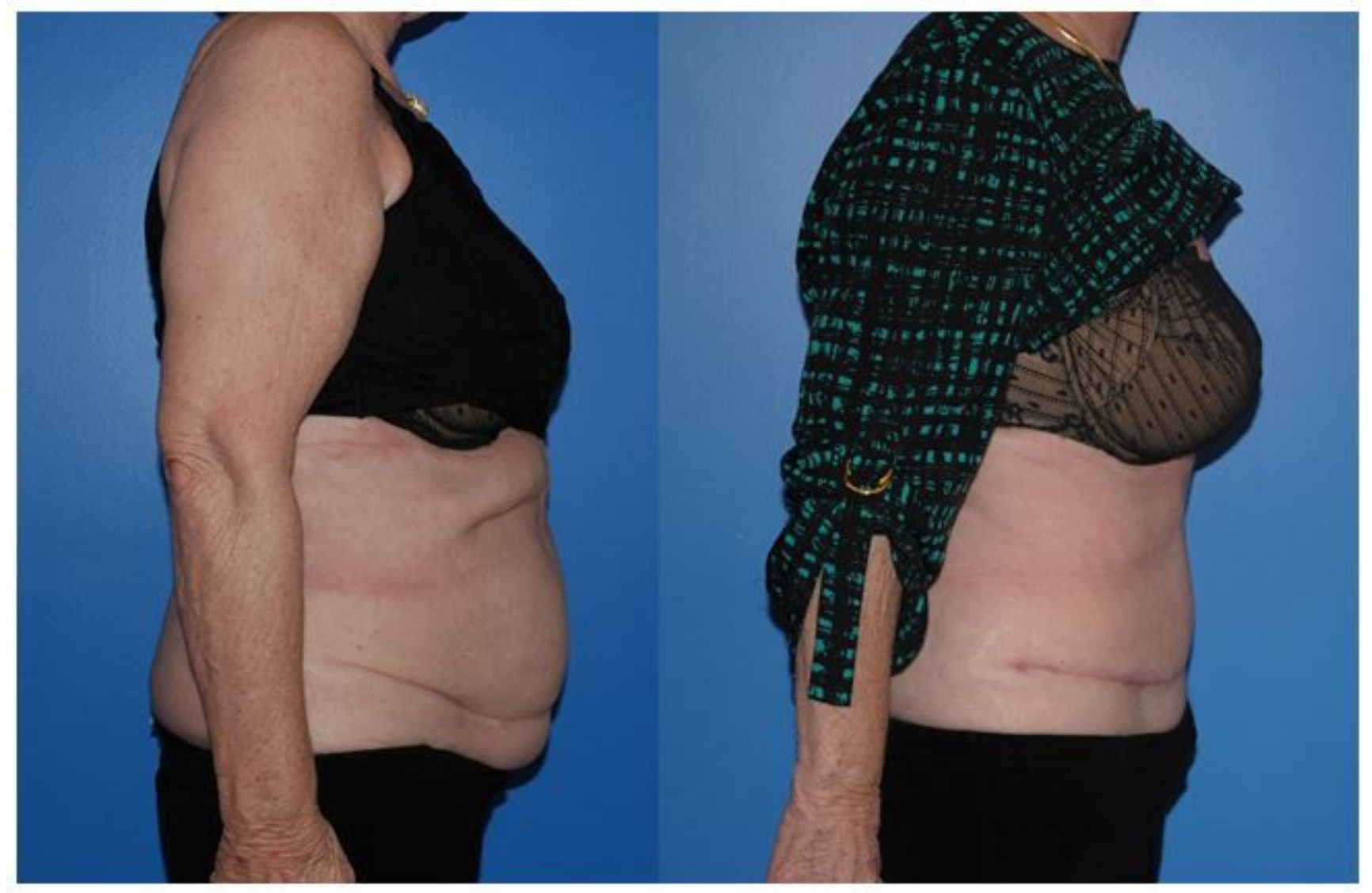

\section{Figure 27}

Ventral hernia with tethered scars and a lower abdominal incision. The previous incisions were joined for access resulting in an inverted T. Caution needs to be used with long superior based flaps as the skin has a high rate of necrosis secondary to dependent venous drainage. 


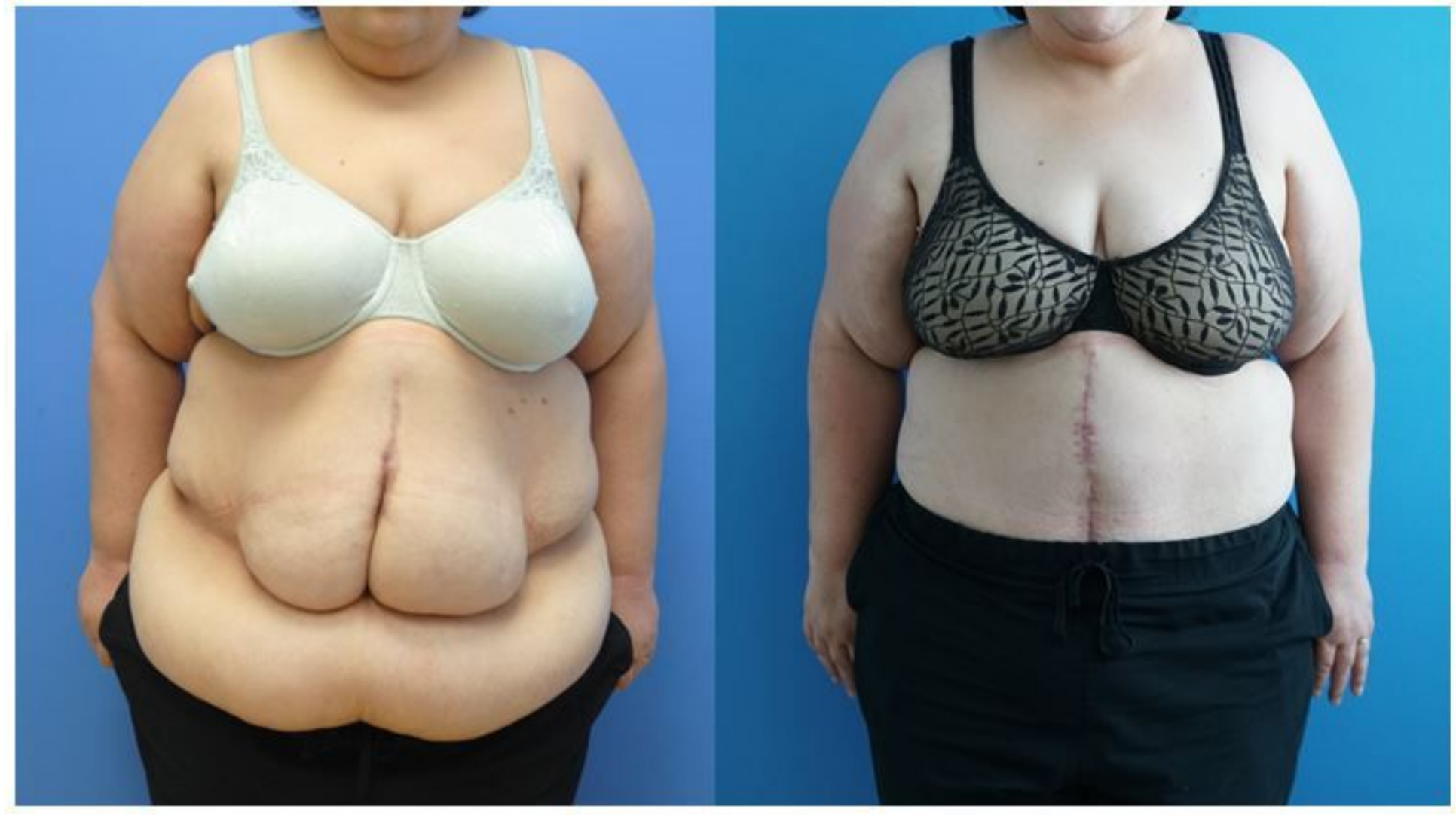

Figure 28

Ventral hernia high BMI with previous wound infection. The hernia was repaired with component separation and repair without mesh given her high BMI. The risk for seroma formation was high after wide undermining and the excess skin and abdominal pannus were excised simultaneously. 


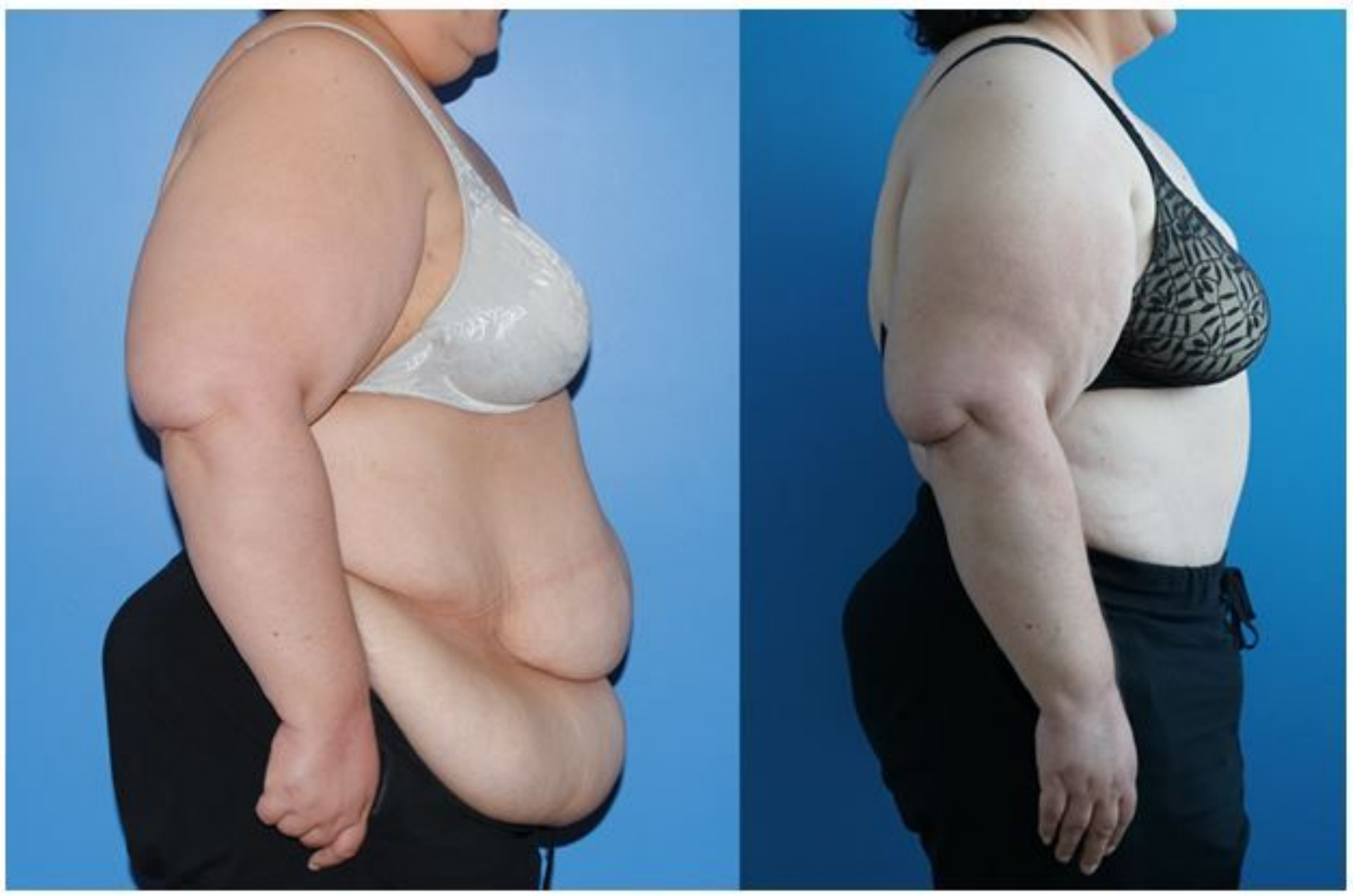

Figure 29

When the superficial epigastric vessels are divided the venous return to the large skin flaps are via the external intercostals in the opposite direction. It is important to counsel patients that there is a high likelihood of abdominal skin necrosis the location of wound sites where T-junctions come together.

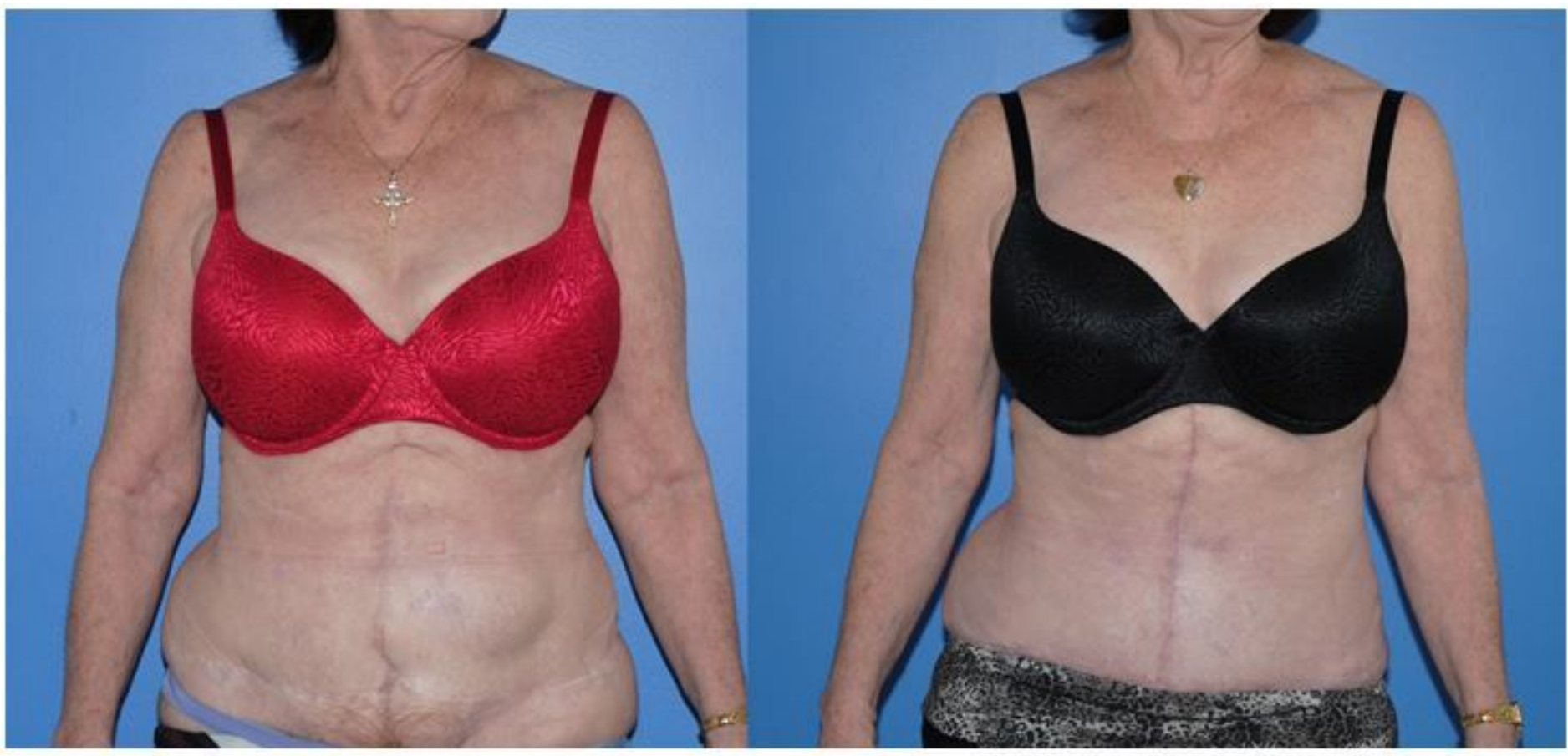

Figure 30 
Hernias at previous colostomy sites typically occur at the location of the rectus sheath. Separating the components and repairing each is important and is an ideal location for the retro-rectus placement of an acellular dermal matrix.

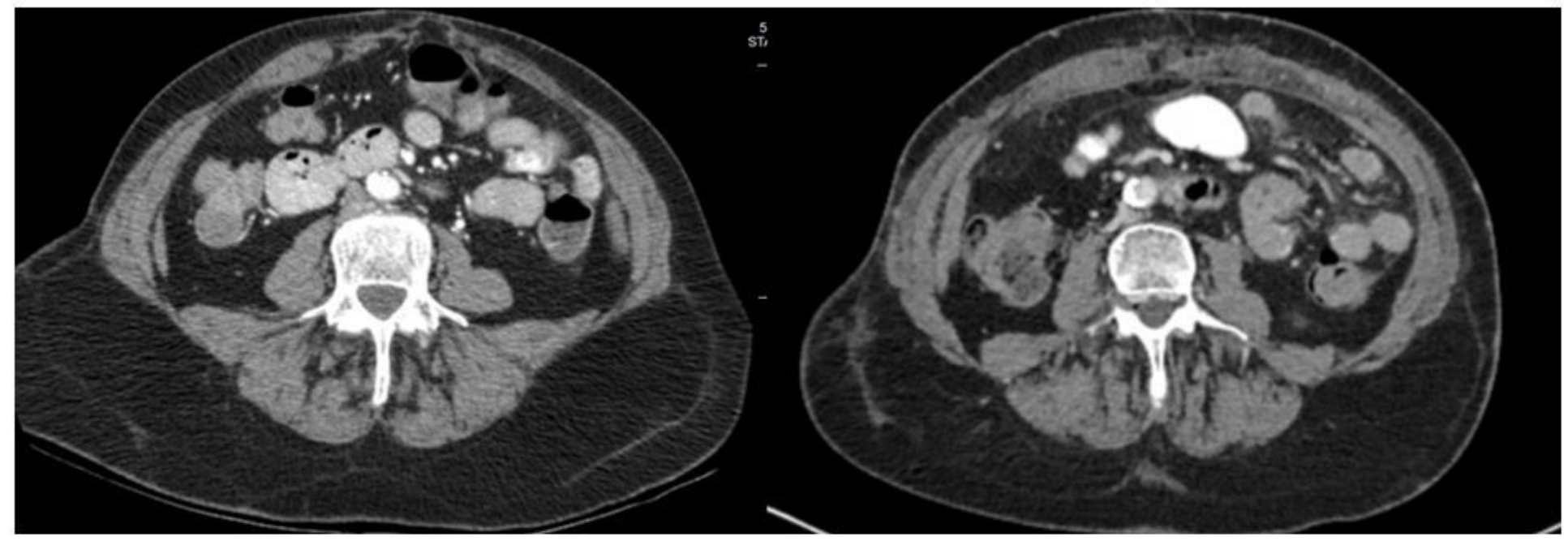

\section{Figure 31}

The segmental innervation of the rectus muscle usually permits excellent muscle apposition and function following repair of the layers of the rectus sheath with the addition of retro-rectus placement of mesh or acellular dermal matrix.

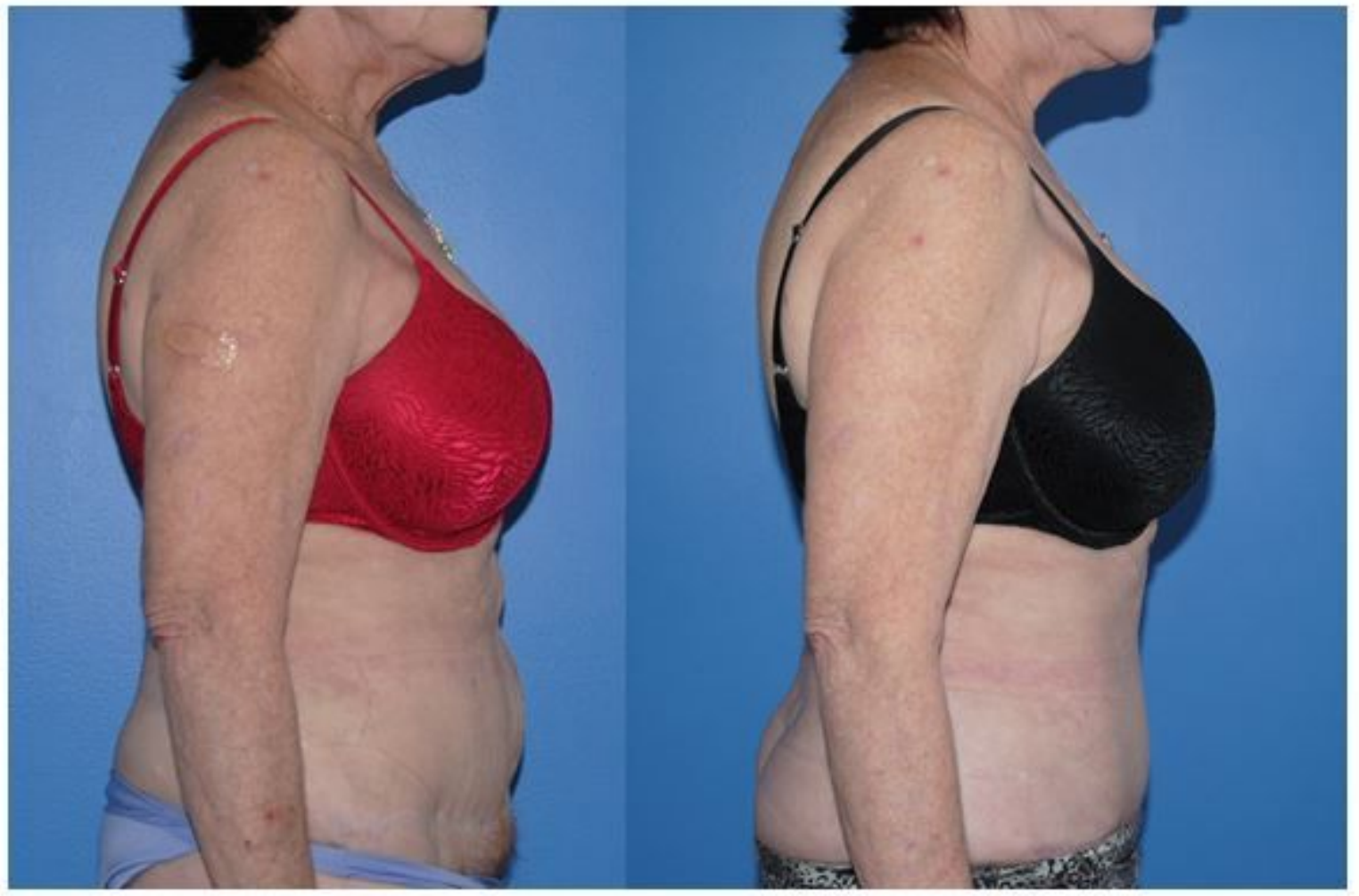

Figure 32 
The segmental innervation of the rectus muscle usually permits excellent muscle apposition and function yielding a stable repair and good aesthetic contour.

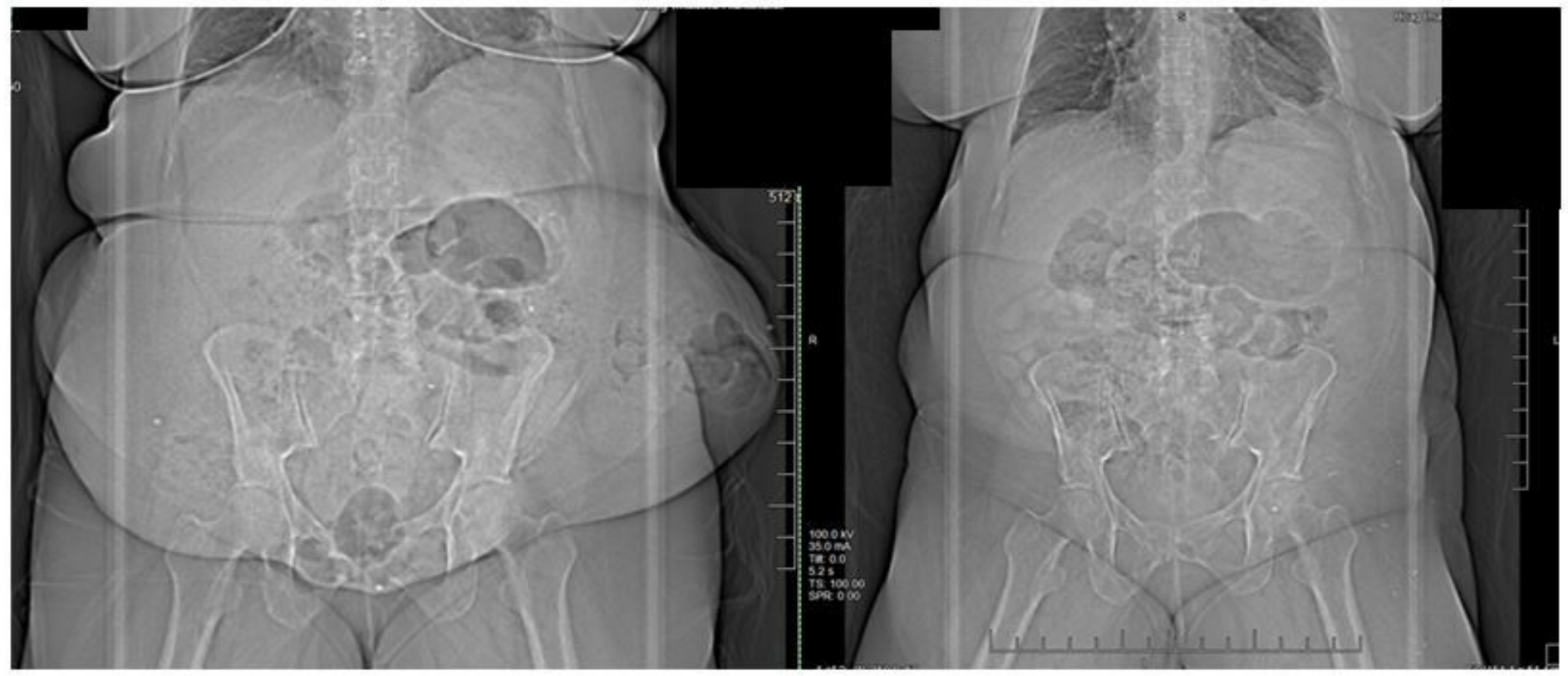

\section{Figure 33}

Control of soft tissue is very important in reconstruction of the abdominal wall. Removal of hernia sacs from subcutaneous tissue can lead to large pockets that can create persistent seromas that can ultimately become infected. Careful attention to and obliteration of dead space is important.

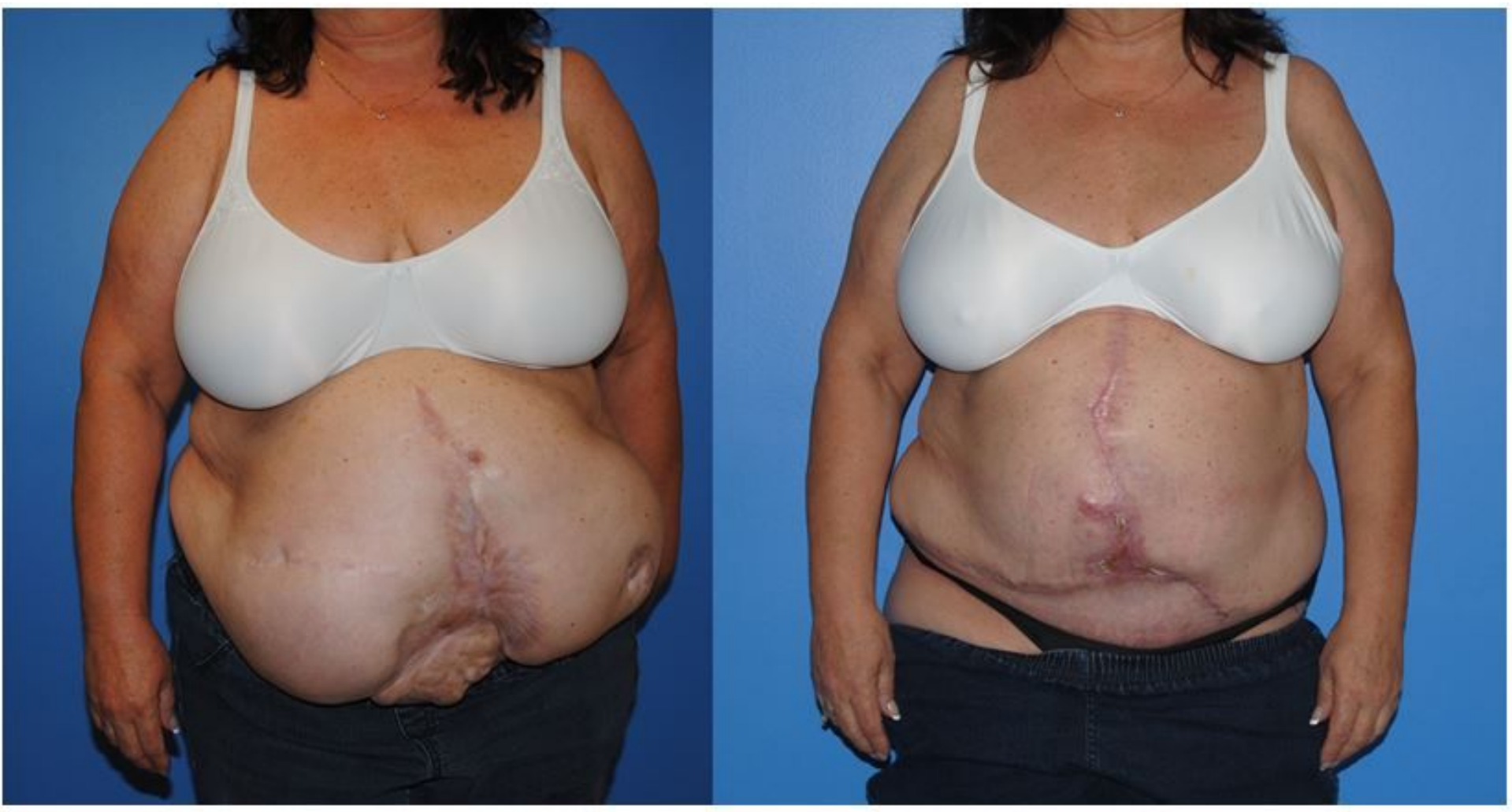

Figure 34 
The patient had a high BMI and an acellular dermal matrix bridge was placed previously during a colon anastomosis. Given her high rate of infection, the abdomen was closed with a tensor fascia lata flap and excision of undermined skin to prevent seroma and infection. The small area of delayed wound healing eventually closed in the absence of mesh.

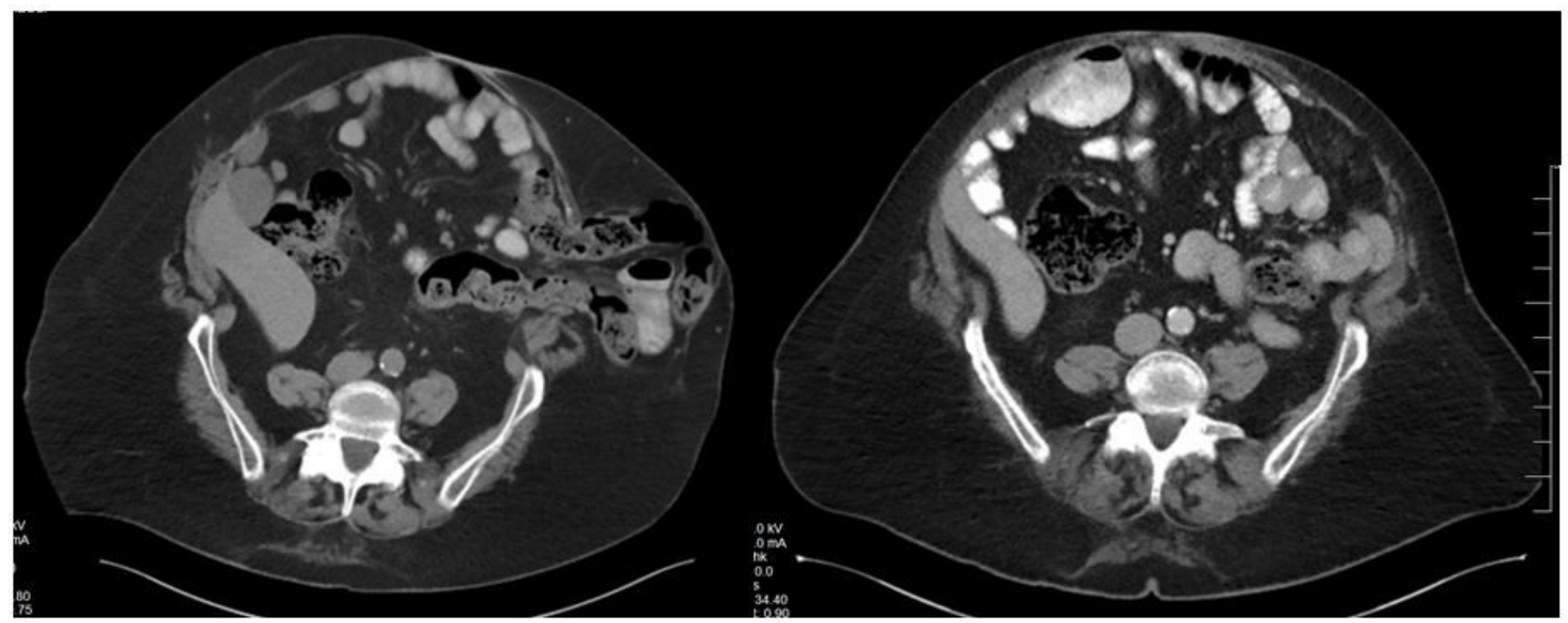

\section{Figure 35}

Control of soft tissue is very important in reconstruction of the abdominal wall. Removal of hernia sacs from subcutaneous tissue can lead to large pockets that can create persistent seromas that can ultimately become infected. Careful attention to and obliteration of dead space is important.

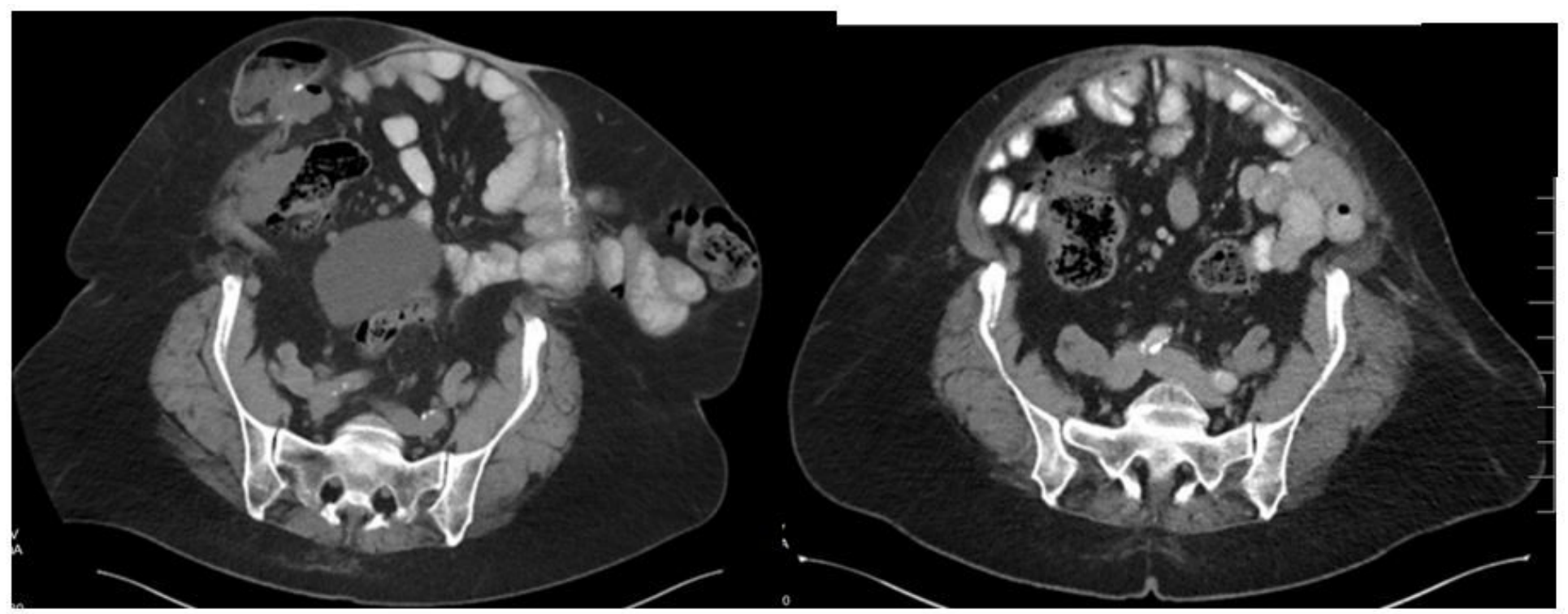

\section{Figure 36}

Control of soft tissue is very important in reconstruction of the abdominal wall. Removal of hernia sacs from subcutaneous tissue can lead to large pockets that can create persistent seromas that can ultimately become infected. Careful attention to and obliteration of dead space is important. 

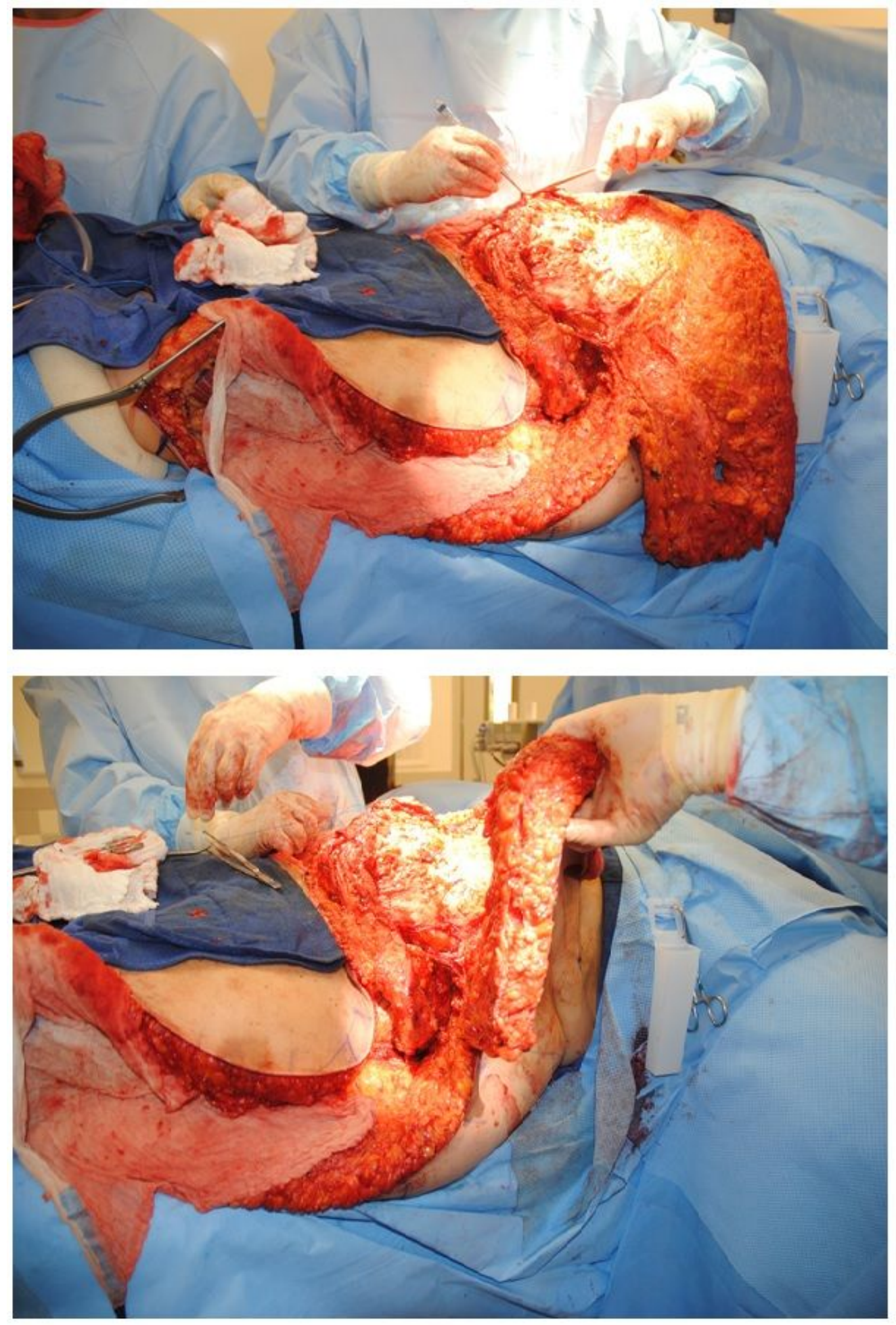

\section{Figure 37}

In patients who mesh is to be avoided, the tensor fascia lata muscle can be dissected and used to repair the anterior abdominal wall. It will prevent adhesions to underlying bowel, placing it in a complete underlay fashion is challenging given the pedicled nature of the flap. In patients who mesh is to be avoided, the tensor fascia lata muscle can be dissected and used to repair the anterior abdominal wall. It 
will prevent adhesions to underlying bowel, placing it in a complete underlay fashion is challenging given the pedicled nature of the flap.

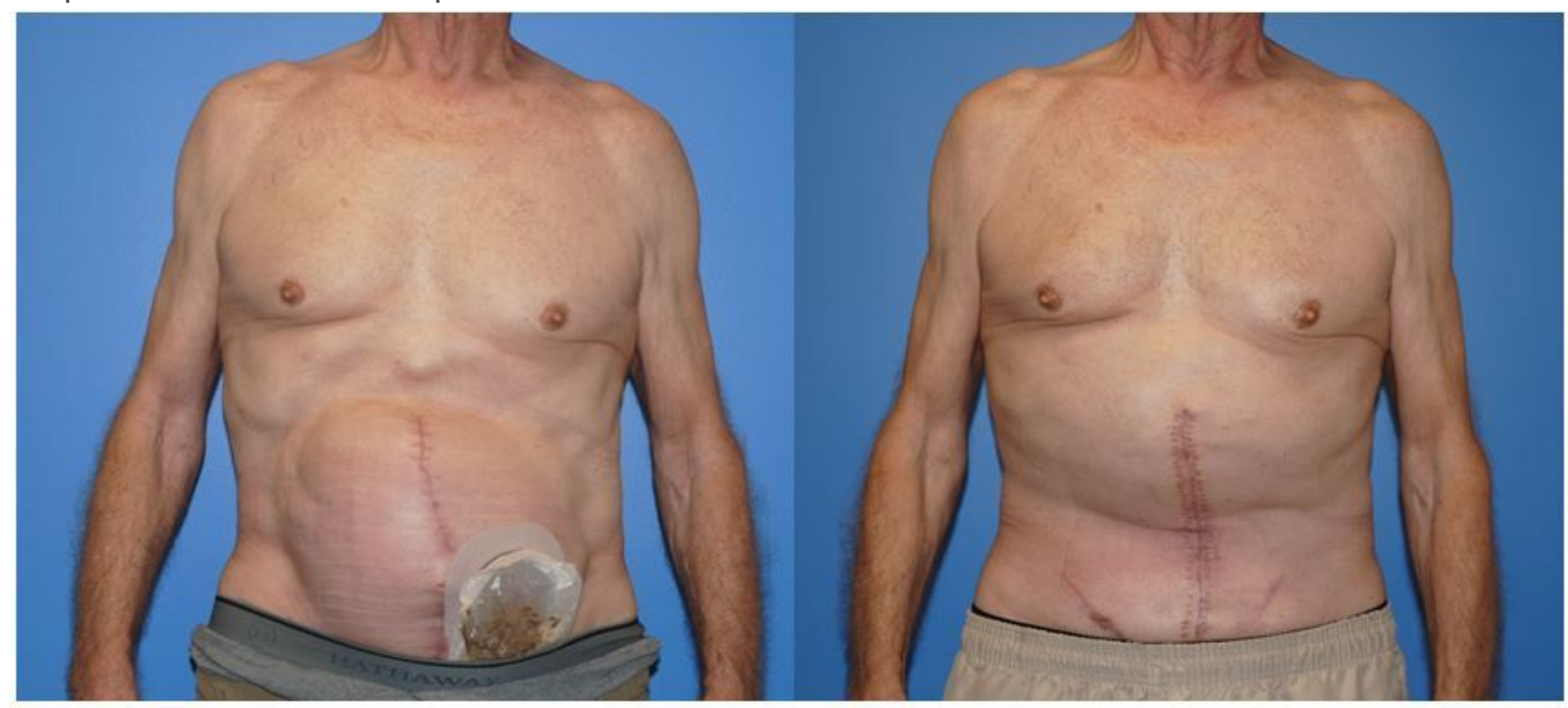

\section{Figure 38}

Large abdominal hernia post colostomy and wished to undergo hernia repair simultaneous with reanastomosis. Component separation with release of the external oblique aponeurosis was completed with underlay of a biologic mesh.

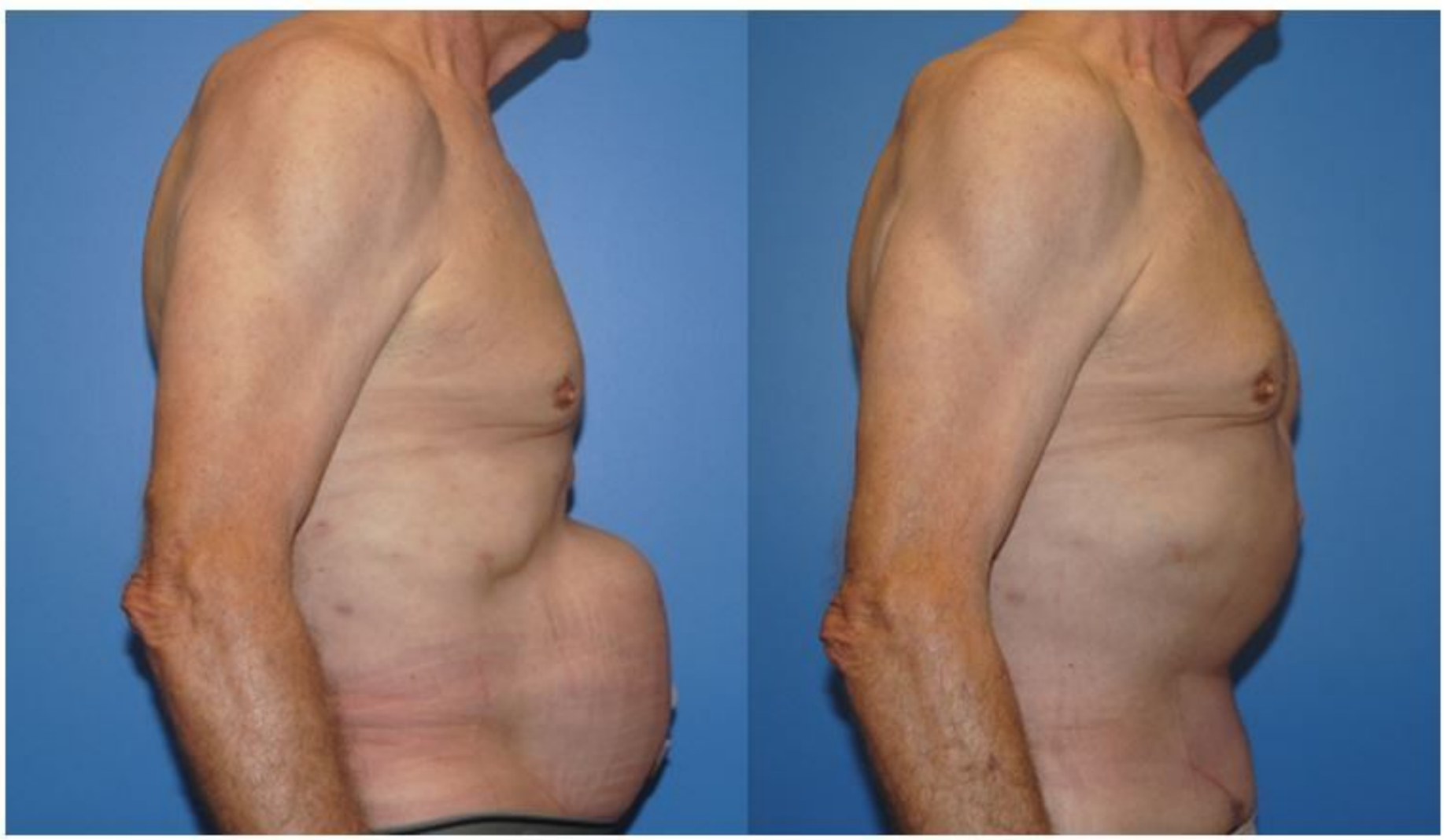




\section{Figure 39}

Biologic mesh is helpful in the setting of colostomy or ileostomy as it is more resistant to infection. Multiple drain placement between the biologic mesh and the fascia and the subcutaneous tissue is important to prevent infection of seromas.

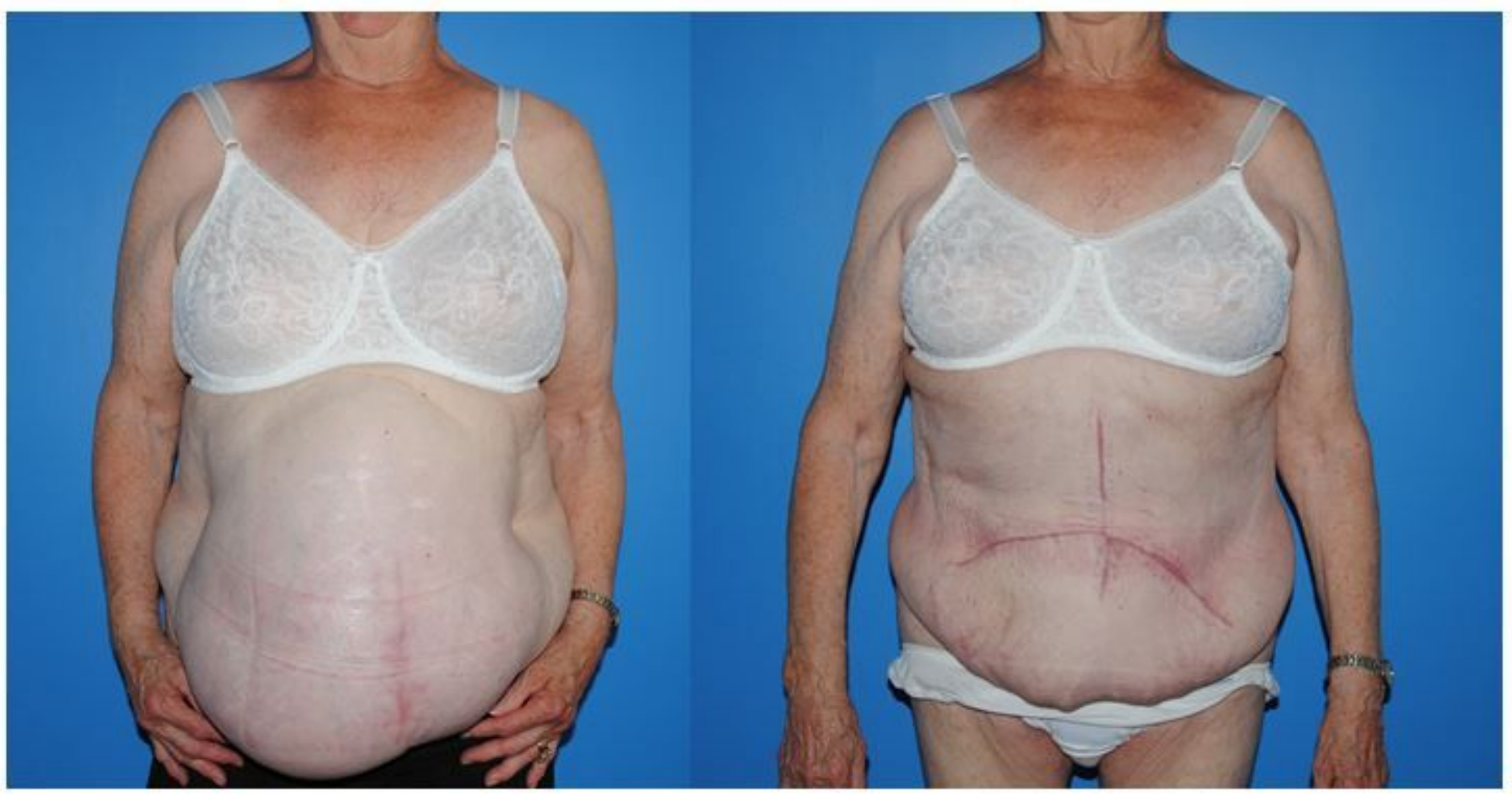

Figure 40

Large ventral hernia with significant amount of abdominal contents outside of domain and in subcutaneous tissue. The intestines expanded the skin. The excess skin was excised in vertical and horizontal direction to close dead space and prevent hernia formation. Care should be taken with excision of external oblique muscle and placement of transfascial sutures. Can lead to transient myoglobinuria and renal insufficiency if baseline nephropathy and dehydration from bowel prep. 


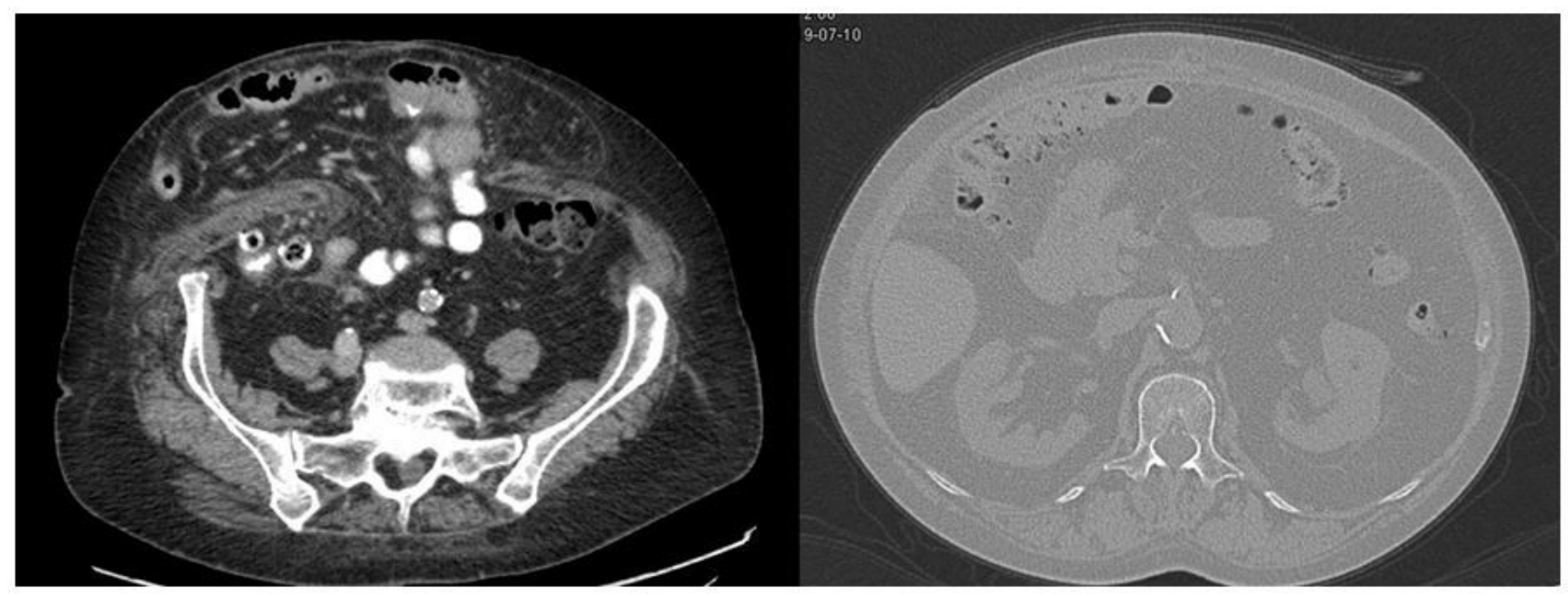

\section{Figure 41}

CT-scan of large ventral hernia with significant amount of abdominal contents outside of domain and in subcutaneous tissue. The intestines expanded the skin. The excess skin was excised in vertical and horizontal direction to close dead space and prevent hernia formation. Care should be taken with excision of external oblique muscle and placement of transfascial sutures. Can lead to transient myoglobinuria and renal insufficiency if baseline nephropathy and dehydration from bowel prep.

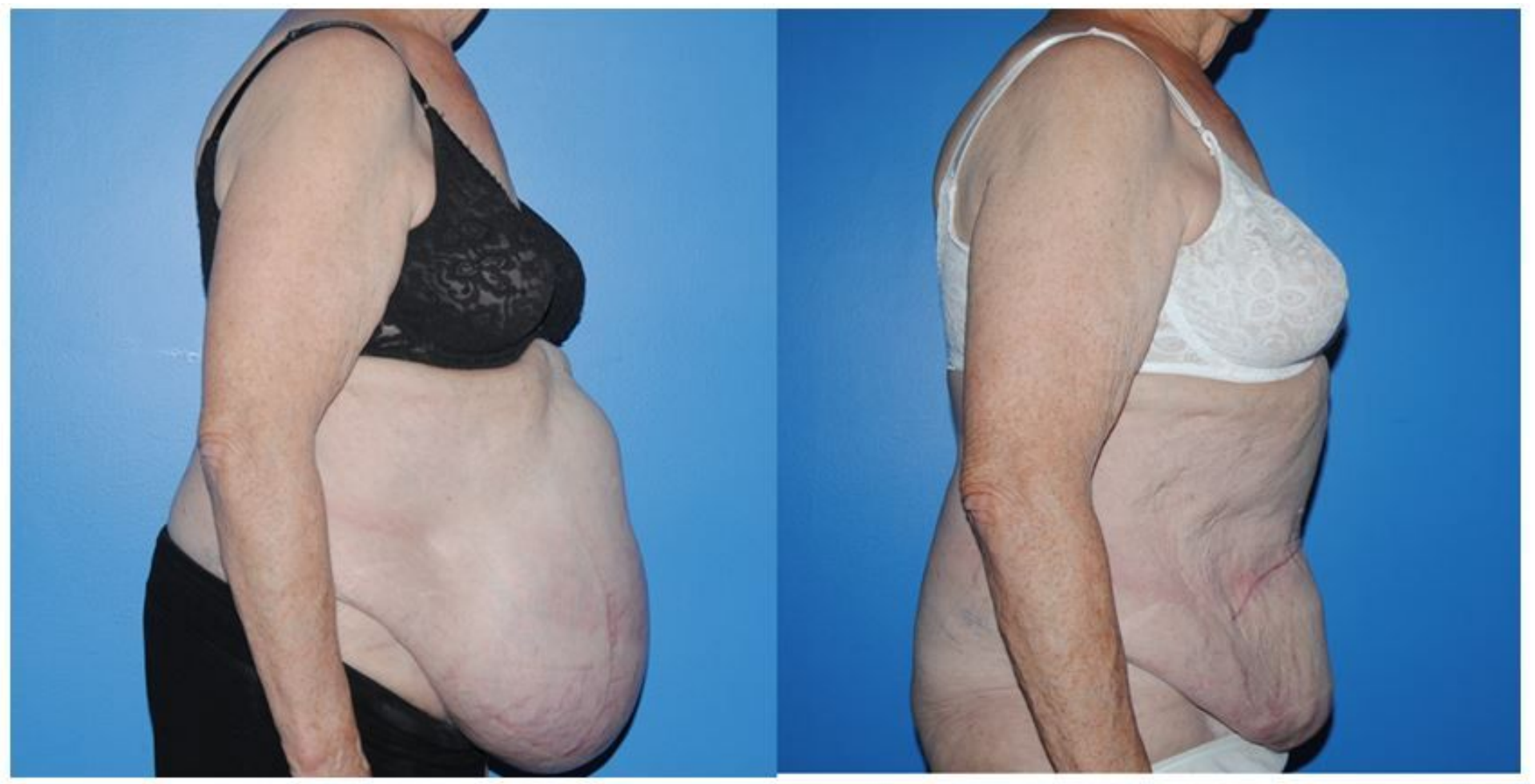

\section{Figure 42}

Large ventral hernia with significant amount of abdominal contents outside of domain and in subcutaneous tissue. The intestines expanded the skin. The excess skin that is expanded inferiorly can 
be elevated based on the superficial epigastric vessels. This can be utilized and move cephalad. Patients will continue to lose weight after abdominal wall reconstruction.

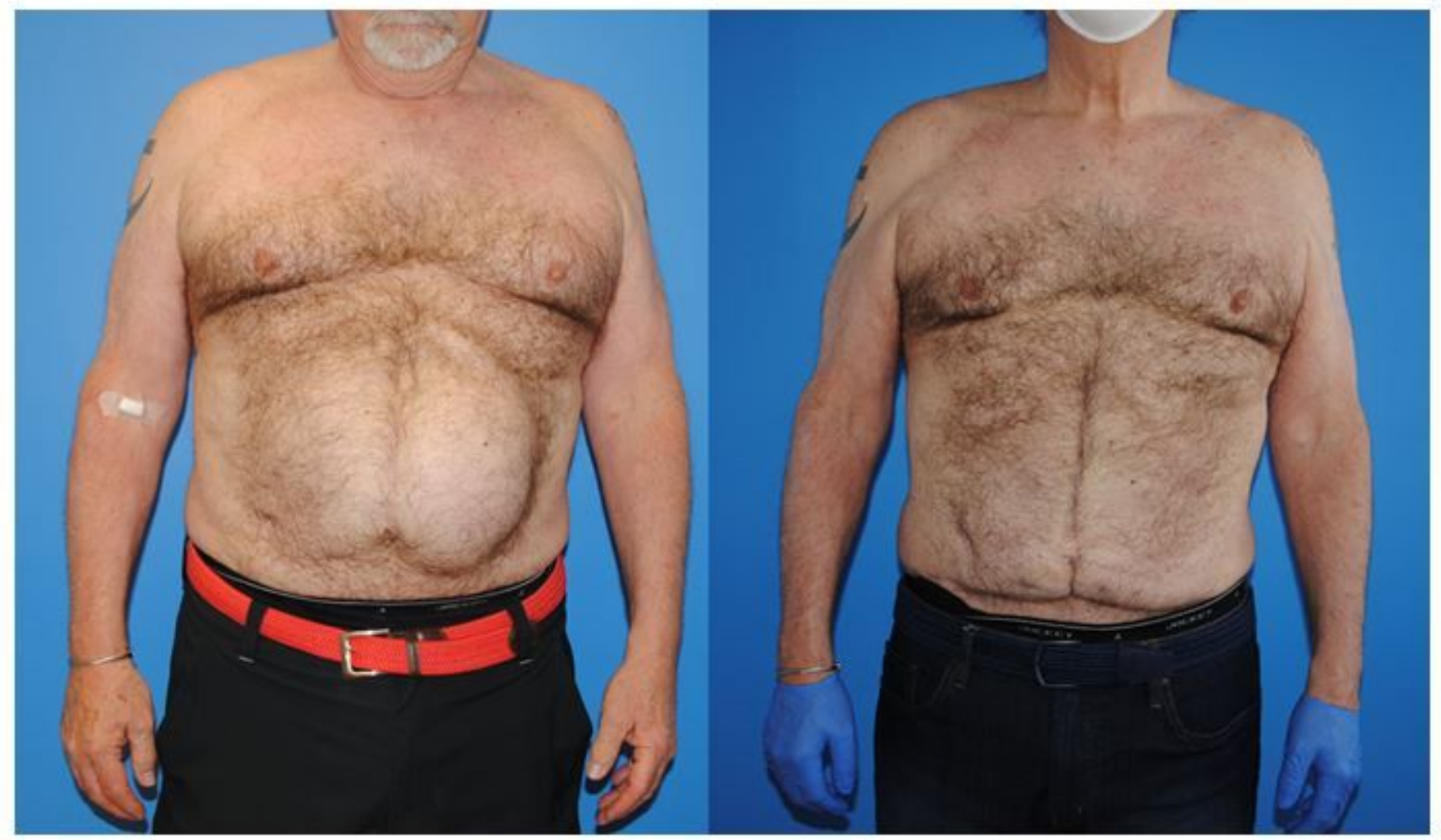

\section{Figure 43}

Large ventral recurrent incisional hernia of abdominal wall. In taller muscular patients, with high BMI, we find that open repair with underlay of extra thick Strattice acellular dermal matrix with transfascial sutures and muscular closure with formation of a dynamic abdominal wall yields excellent repair. The primary author has attempted complete muscular closure without underlay with load sharing and it has resulted in recurrences.

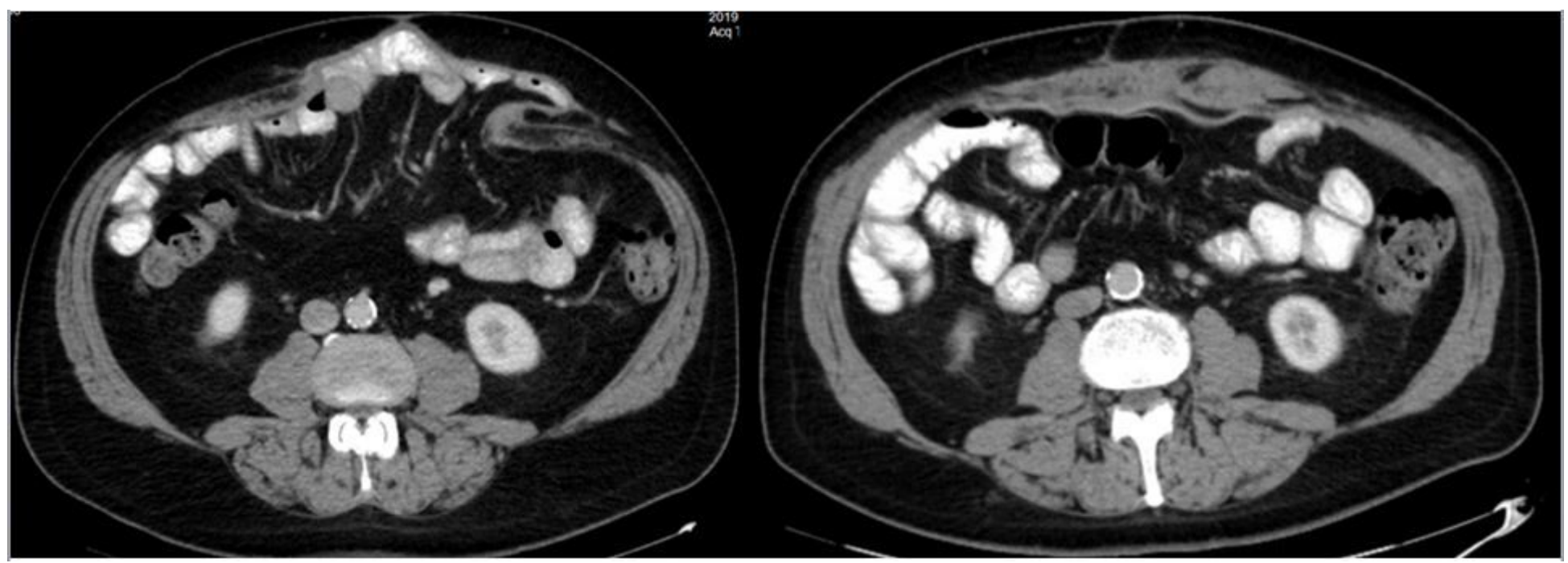

Figure 44 
The muscular patients with high (BMI - 28.7) can be as concerning for repair as the obese patient with the high BMI. Often these patients may return to working out or manual labor and develop early recurrence. In these patients we wait until six weeks before beginning physical therapy. When therapy starts at 6 weeks, they begin with 2 weeks of isometric core exercises.

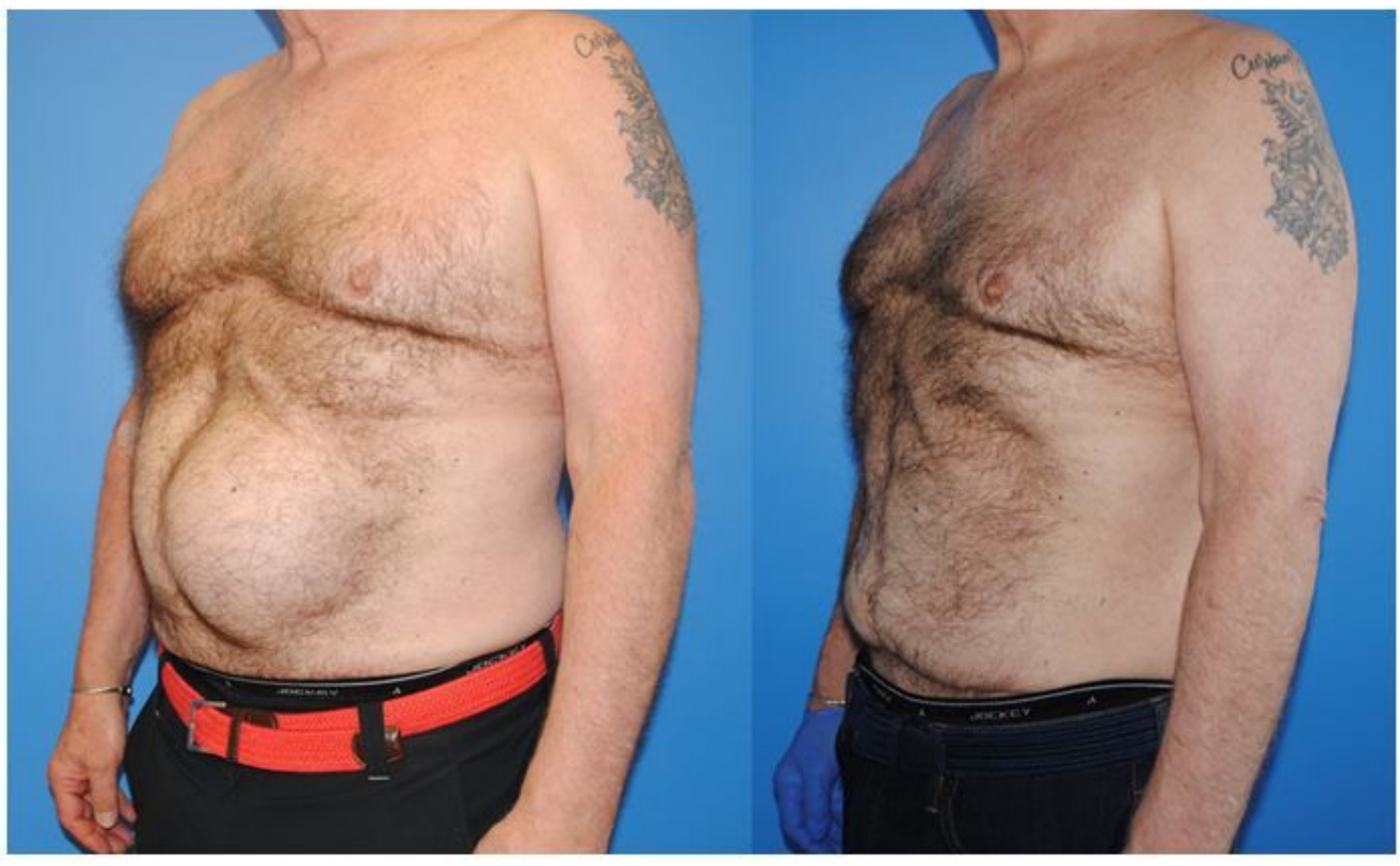

\section{Figure 45}

The initiation of a high protein diet for six to eight weeks prior to the abdominal wall reconstruction can help patients lose weight and also decrease the amount of mesenteric fat. This not only facilitates abdominal wall closure, but also prevents future recurrences. Patients often continue the high protein diet after and continue to lose weight. The decrease in domain also results in early satiety which can facilitate weight loss. 


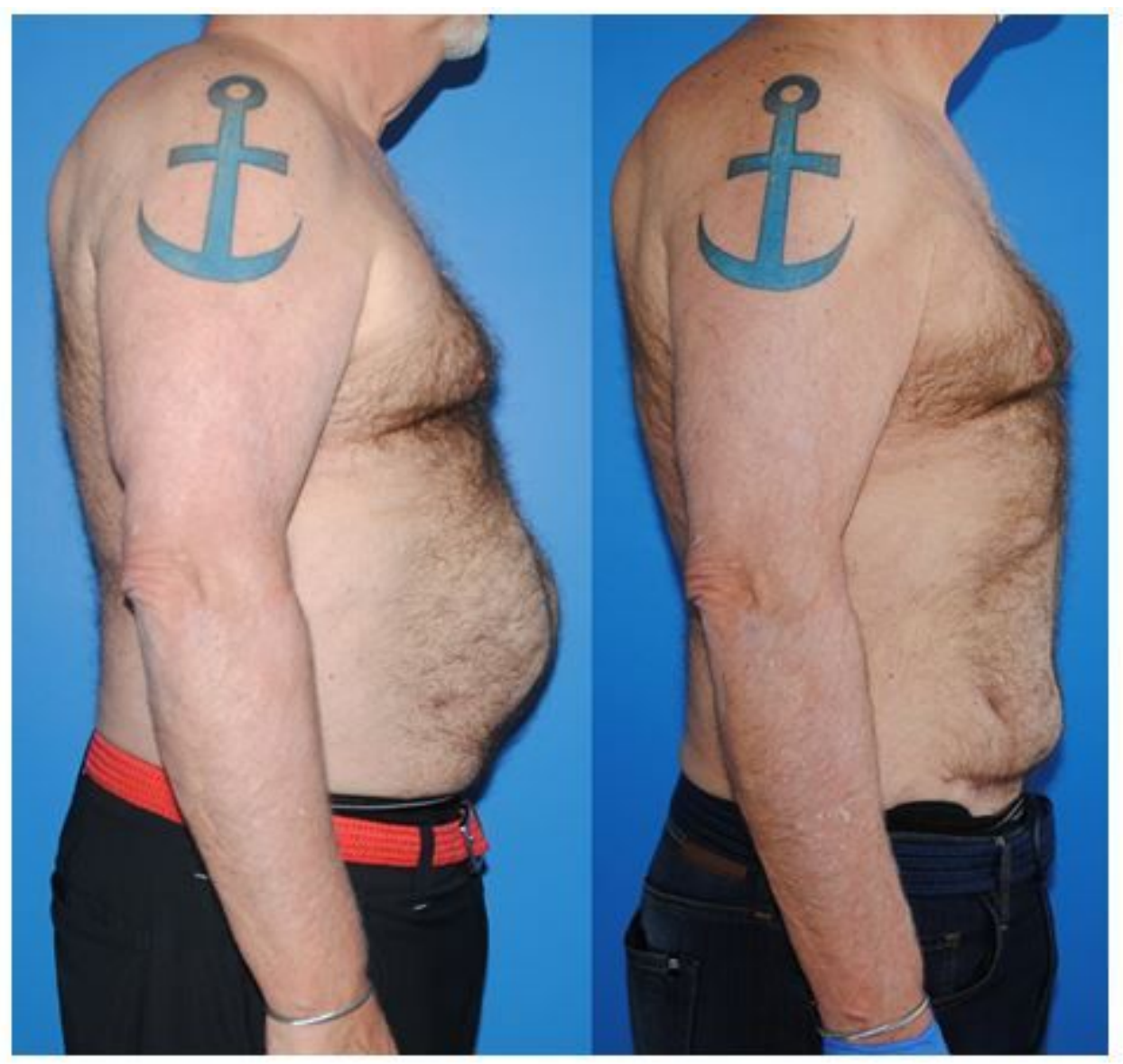

\section{Figure 46}

In taller muscular patients we find that open repair with underlay of Strattice acellular dermal matrix with transfascial sutures and muscular closure with formation of a dynamic abdominal wall yields excellent repair. The primary author has attempted complete muscular closure without underlay with load sharing and it has resulted in recurrences. 


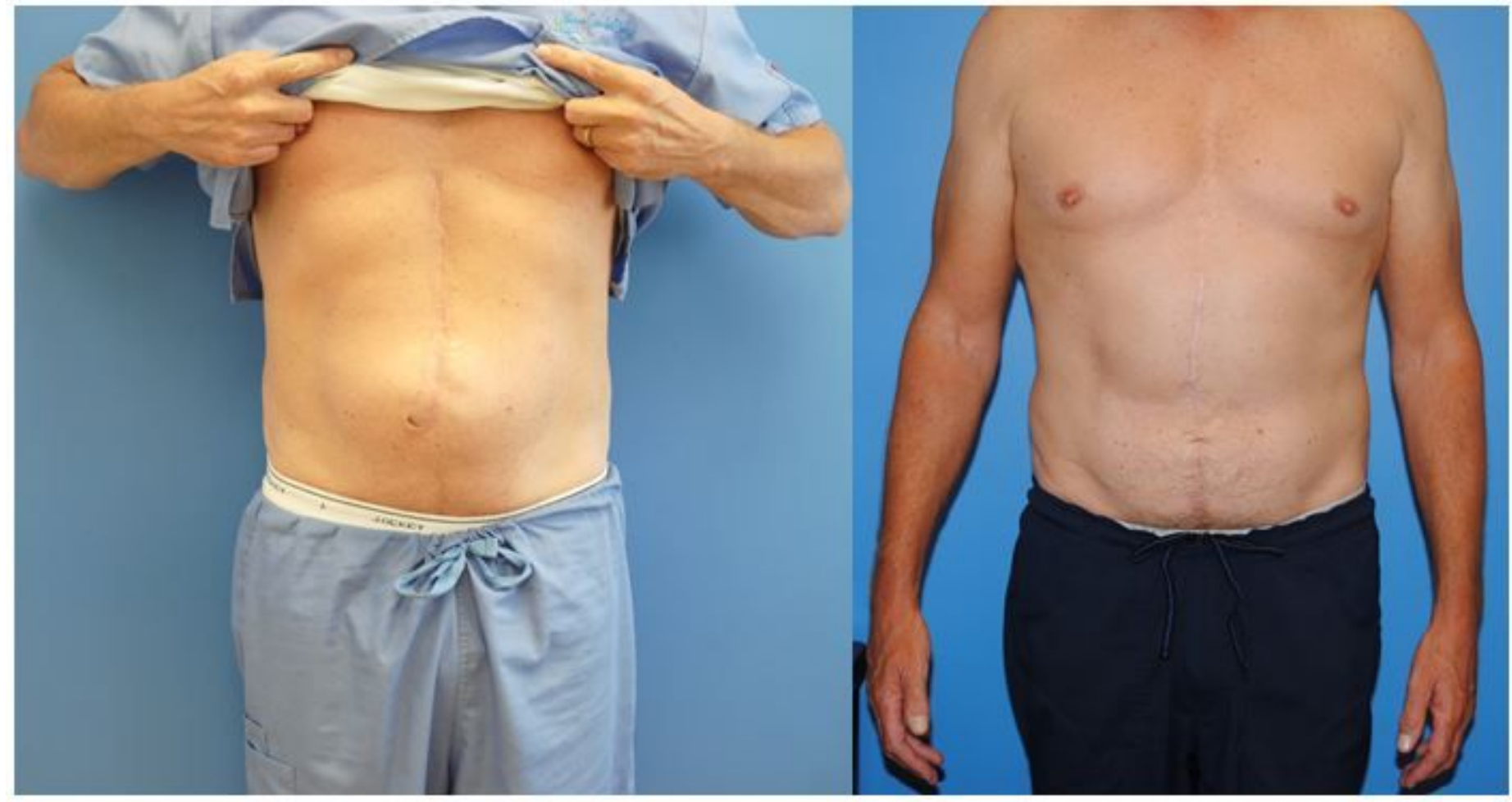

Figure 47

Abdominal wall reconstruction in the tall patient with a high BMI should be completed with a load sharing approach. The primary author has attempted complete muscular closure without underlay mesh and component separation and it has resulted in recurrences. In patients who want to avoid mesh, the biologic Strattice extra thick is helpful for load sharing repair.

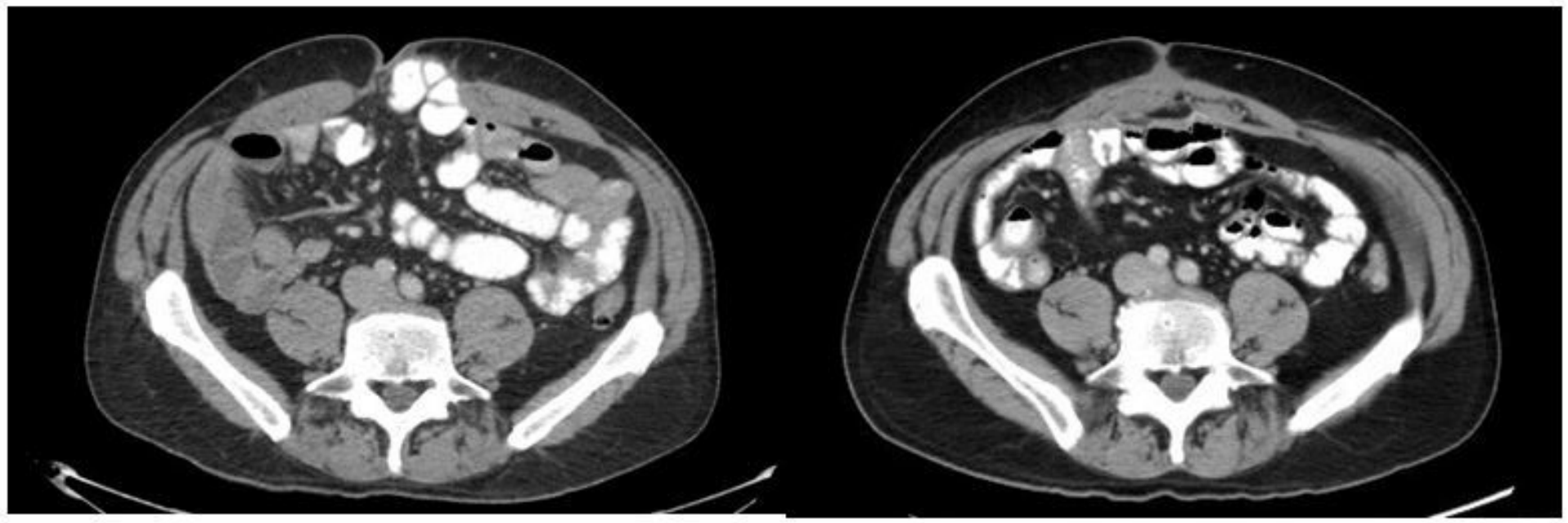

\section{Figure 48}

Abdominal wall reconstruction in the tall patient with a high BMI should be completed with a load sharing approach. The primary author has attempted complete muscular closure without underlay mesh and 
component separation and it has resulted in recurrences. In patients who want to avoid mesh, the biologic Strattice extra thick is helpful for load sharing repair.

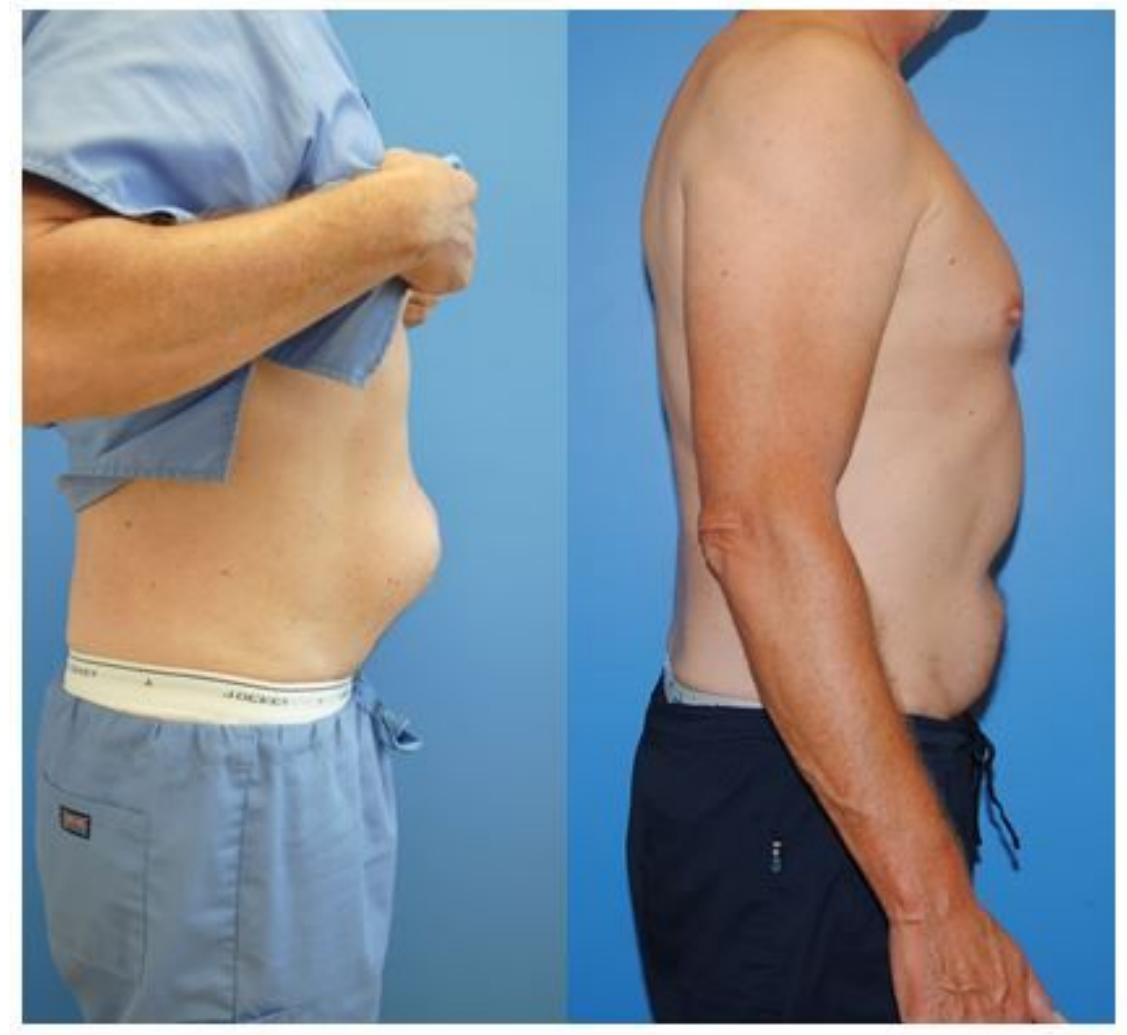

\section{Figure 49}

Abdominal wall reconstruction in the tall patient with a high BMI should be completed with a load sharing approach. The primary author has attempted complete muscular closure without underlay mesh and component separation and it has resulted in recurrences. In patients who want to avoid mesh, the biologic Strattice extra thick is helpful for load sharing repair. 


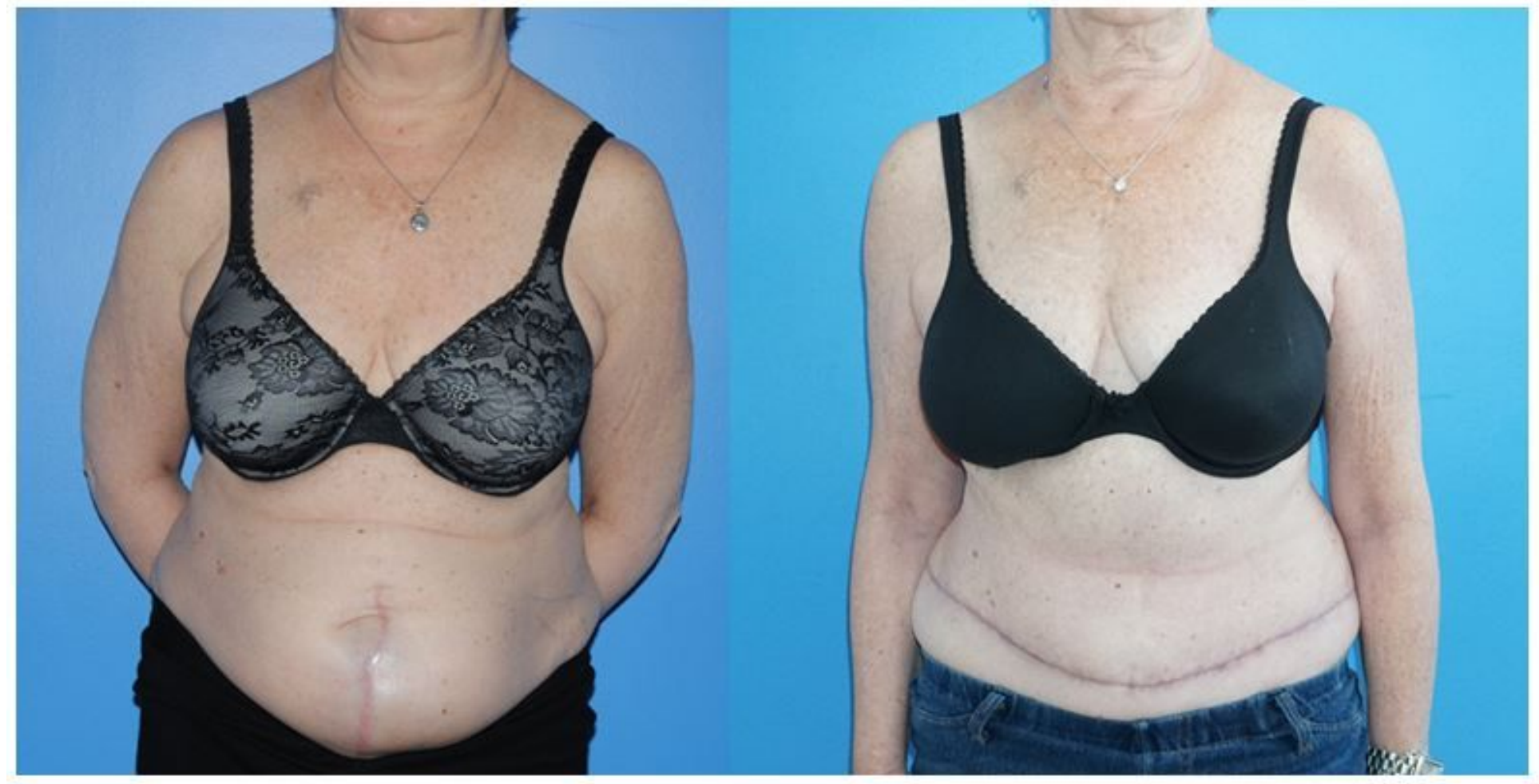

\section{Figure 50}

There are often patients who choose to avoid both synthetic and biologic mesh. In these patients, the anterior component separation repair works well, although patient selection is important for success. The best repairs are in those with a low BMI and who are not avid exercise enthusiasts.

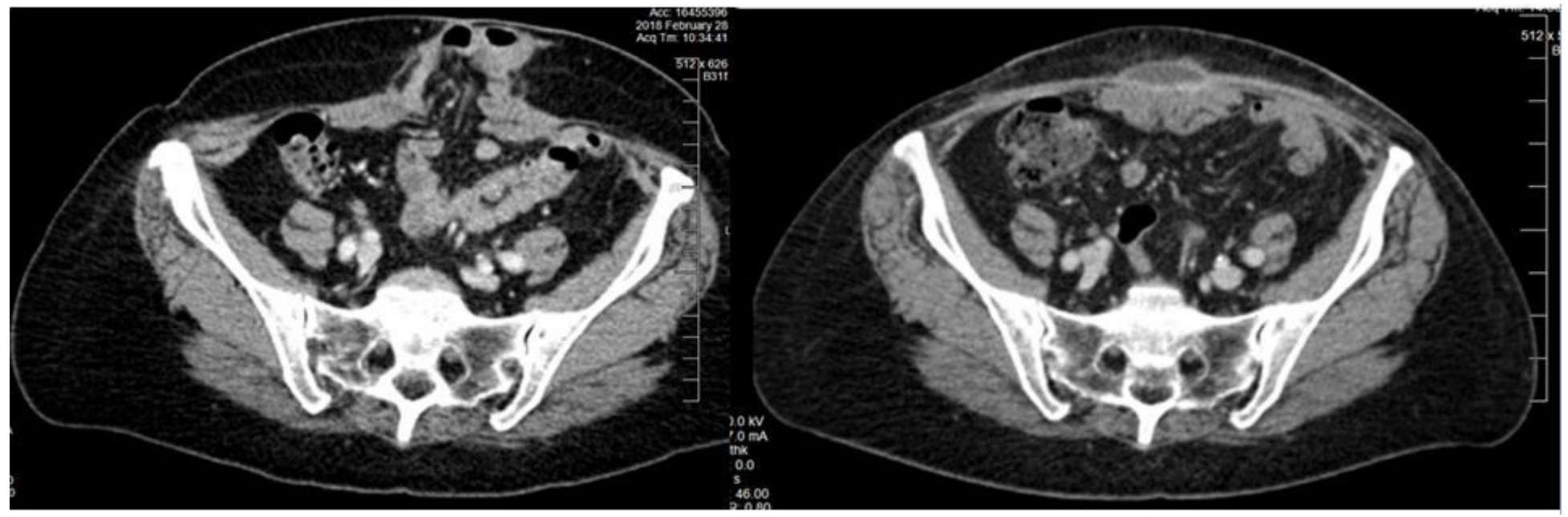

\section{Figure 51}

Repair without synthetic or bioprosthetic mesh involves careful repair of the midline hernia defect and also includes a second layer of rectus muscle repair. Small seromas or fluid collections anterior to the fascia are common after removal of drains as patient activity increases.

\section{Supplementary Files}


This is a list of supplementary files associated with this preprint. Click to download.

- AuthorizationofReleaseofPatientPhotograph1.pdf

- Supplement.pptx 